\title{
Homological Lagrangian monodromy
}

\author{
SHENGDA HU \\ FRANÇOIS LALONDE \\ RÉMI LECLERCQ
}

\begin{abstract}
We show that the Hamiltonian Lagrangian monodromy group, in its homological version, is trivial for any weakly exact Lagrangian submanifold of a symplectic manifold. The proof relies on a sheaf approach to Floer homology given by a relative Seidel morphism.
\end{abstract}

53D12, 53D40; 53C15, 53D45, 57R58, 57S05, 58B20

\section{Introduction}

Given a Lagrangian submanifold $L \subset M$ embedded in a symplectic manifold $M$, it is natural to consider the subgroup $\mathcal{G} \subset \operatorname{Diff}(L)$ consisting of all diffeomorphisms of $L$ that can be obtained as the time-one map of a Hamiltonian (that is, exact) Lagrangian isotopy $\phi_{t \in[0,1]}: L \rightarrow M$ that starts at $t=0$ at the identity map on $L$ and ends at $t=1$ at a diffeomorphism that preserves $L$. In other words, if one denotes by $\operatorname{Ham}_{L}(M) \subset \operatorname{Ham}(M)$ the subgroup of the group of Hamiltonian diffeomorphisms of $M$ consisting of the diffeomorphisms $g$ satisfying $g(L)=L$, the group $\mathcal{G}$ is then the image of the homomorphism

$$
\operatorname{Ham}_{L}(M) \rightarrow \operatorname{Diff}(L)
$$

that assigns to each diffeomorphism $g \in \operatorname{Ham}_{L}(M)$ its restriction to $L$. Denoting by $R$ any given ring, the homological Hamiltonian Lagrangian monodromy problem is the study of the subgroup $\mathcal{G}_{*, R}$ of $\operatorname{Aut}\left(H_{*}(L ; R)\right)$ defined as the image of $\mathcal{G}$ under the map that assigns to each diffeomorphism its action on homology (we will often assume that $R$ is given and will omit it in our notations; we will also omit the word "exact" since we will work with Hamiltonian isotopies only in this paper).

The homological Lagrangian monodromy group $\mathcal{G}_{*, R}$ is an invariant attached to each exact Lagrange isotopy class of a given Lagrangian submanifold. It is therefore of prime importance in the Lagrangian knot problem.

To our knowledge, this group has been studied only very recently by Mei-Lin Yau [13], in the two cases of the standard monotone 2-torus and of the Chekanov torus, both 
living in $\mathbb{R}^{4}$, using soft methods in a clever way. Let $\Theta_{t \in[0,1]}$ be the standard oneparameter family of elements of $S O(2)$ starting at the identity anti-clockwise and ending at the rotation by angle $\pi / 2$. By the standard inclusion $S O(2) \subset U(2)$, the same path can be considered as a path of elements of $U(2)$ and it clearly restricts to an exact isotopy of the standard torus $T_{a, a}=S^{1}(a) \times S^{1}(a) \subset \mathbb{C} \times \mathbb{C}$ (here the number in parentheses denotes the area of the circle) whose endpoint at $t=1$ permutes the two standard generators of $H_{1}\left(T_{a, a} ; \mathbb{Z}\right)$. The main result of [13] is that this induces the only non-trivial element of $\mathcal{G}_{*, \mathbb{Z}}$, and thus $\mathcal{G}_{*, \mathbb{Z}}=\mathbb{Z}_{2}$ for the standard torus. M-L Yau also shows that the same result holds for the Chekanov 2-torus of $\mathbb{R}^{4}$. Moreover she shows that the intrinsic spectral (and symplectic) invariants attached to the non-trivial element of $\mathcal{G}_{*, \mathbb{Z}}$ in each of these two cases are different, so that this provides another proof of the fact that the standard 2-torus is not exact Lagrange isotopic to the Chekanov 2-torus.

The other extreme case is the one of a closed exact Lagrangian submanifold $L$ in a cotangent bundle $T^{*} V$. A famous conjecture states that $L$ should then be Hamiltonian isotopic to the zero section. If this conjecture is true, then obviously the group $\mathcal{G}_{*}$ is trivial for all coefficients, that is to say it consists of the identity only. A homological version of this conjecture has been proved by Fukaya-Seidel-Smith [7]: they have indeed shown that if $V$ is simply connected, then an exact Lagrangian embedding with vanishing Maslov class of a spin manifold $L$ in $T^{*} V$ must project to a map $L \rightarrow V$ inducing an isomorphism in homology over $\mathbb{Q}$. Thus, under these hypotheses, the group $\mathcal{G}_{*, \mathbb{Q}}$ is clearly trivial.

The main goal of this paper is to prove the fundamental result that, when $L$ is a weakly exact Lagrangian submanifold of a symplectic manifold $M$, and under certain natural conditions on $L$ only related to the choice of the coefficients ring, this still holds, that is to say the group $\mathcal{G}_{*}$ is trivial. Thus, at least as far as the group $\mathcal{G}_{*}$ is concerned, a weakly exact Lagrangian submanifold behaves like the zero section of a cotangent bundle. The additional natural conditions to which we referred are the usual conditions under which the Floer homology over $R$ is well-defined. We recall that, by definition, $L$ is weakly exact if the following map is identically 0 :

$$
I_{\omega}: \pi_{2}(M, L) \rightarrow \mathbb{R}: \beta \mapsto \int_{\beta} \omega
$$

Obviously, this implies that $M$ is symplectically aspherical, that is, $I_{\omega}$ vanishes on $\pi_{2}(M)$.

Our main result is the following: 
Theorem 1.1 Let $(M, \omega)$ be a symplectic manifold and $L \subset M$ a closed weakly exact Lagrangian submanifold. Let $g_{t \in[0,1]}$ be a Hamiltonian diffeotopy of $M$ starting at the identity and ending at a diffeomorphism preserving $L$. Let $f:=\left.g_{1}\right|_{L}$, then the induced map on homology $f_{*}: H_{*}\left(L ; \mathbb{Z}_{2}\right) \rightarrow H_{*}\left(L ; \mathbb{Z}_{2}\right)$ is the identity.

Remark 1.2 In this theorem, instead of homology with coefficients in $\mathbb{Z}_{2}$, we may use $\mathbb{Z}$ or $\mathbb{Q}$ as coefficient ring as well. In these cases, we require $L$ to be relatively spin with a relatively spin structure, and $g_{1}$ to preserve the relatively spin structure. For the simplicity of statements, we use $\mathbb{Z}_{2}$-coefficients in the main text, while discuss the $\mathbb{Z}$ or $\mathbb{Q}$-coefficients in the appendix.

A priori, one could try to prove this statement by using the invariance of the Floer homology along the flow induced by $g_{t \in[0,1]}$ to extract the information on $\mathcal{G}_{*}$, a bit like what one would do using the sheaf of Floer homologies of a given Lagrangian submanifold induced by some Lagrangian fibration. This paper shows how to make sense of this idea. Indeed, our approach relies on the relative Seidel morphism, introduced in $\mathrm{Hu}$-Lalonde [8], associated to a Hamiltonian path $g_{t \in[0,1]}$ with $g_{1} \in \operatorname{Ham}_{L}(M)$, and on the equivalence between two versions of this morphism, one given in analytic terms and the other in geometric terms. The geometric version of this morphism can be considered as the right set up for an implementation of the above "sheaf approach" to the proof of our theorem.

The idea of the proof of the theorem is to first consider the fibration $L \hookrightarrow N \rightarrow S^{1}$ over $S^{1}$ induced by the restriction to $L$ of the path $g_{t \in[0,1]}$ of Hamiltonian diffeomorphisms. We then get the Wang long exact sequence

$$
\cdots \rightarrow H_{q+1}(L) \stackrel{i_{*}}{\rightarrow} H_{q+1}(N) \rightarrow H_{q}(L) \stackrel{f_{*}-\mathrm{id}}{\longrightarrow} H_{q}(L) \stackrel{i_{*}}{\rightarrow} H_{q}(N) \rightarrow \cdots
$$

We must therefore show that

$$
i_{*}: H_{*}(L) \rightarrow H_{*}(N)
$$

is injective. The point is that this fibration constitutes the boundary condition of a Dirichlet problem for the $\bar{\partial}$-operator. Indeed, the path $g_{t \in[0,1]}$ naturally gives rise to a relative fibration $(P, N)$ over $\left(D^{2}, S^{1}\right)$ with fiber $(M, L)$ and the linearity of the relative Gromov-Witten invariants will lead us to a proof of the injectivity of $i_{*}: H_{*}(L) \rightarrow H_{*}(N)$. This scheme of proof can be considered as the relative Lagrangian version of the main theorem in Lalonde-McDuff-Polterovich [9]. This will be shown in Section 3 using our geometric Seidel map defined in the next section. In the last section of the paper, we give another proof of our main theorem which is 
more algebraic and simpler, but less geometric. It is possible that this second proof could also be derived using the main results in Fukaya-Oh-Ohta-Ono [6, Section 22].

Note that this theorem, in its contrapositive version, provides an obstruction to the extension of a given diffeomorphism $f: L \rightarrow L$ to a Hamiltonian diffeomorphism of the ambient symplectic manifold: more precisely, if $L$ is weakly exact and $f: L \rightarrow L$ induces a map not equal to the identity on say $H_{*}\left(L ; \mathbb{Z}_{2}\right)$, then it cannot be extended to a Hamiltonian diffeomorphism of $M$. Obviously, because a Hamiltonian diffeomorphism is isotopic to the identity, the statement of the theorem is interesting only when $H_{*}(L)$ does not inject in $H_{*}(M)$. There are plenty of closed weakly exact manifolds whose homologies do not inject in the homology of the ambient manifold. The simplest example is the one of a simple closed curve of a closed Riemann surface that bounds homologically but not homotopically, and product of these examples. A less trivial example is the following: consider the quotient $Q$ of $T^{2} \times \mathbb{R}$ by the linear map $(x, y, z) \mapsto(x+y, y, z+1)$ where $x, y$ are the coordinates on $T^{2}$ and $z$ on $\mathbb{R}$. Then $(d x-z d y) \wedge d z$ descends to a form on $Q$. Let $t$ be the coordinate on $S^{1}$. The form $\omega=(d x-z d y) \wedge d z+d y \wedge d t$ is then well defined and is symplectic on $Q \times S^{1}$. Moreover, $T^{2}$ is Lagrangian. The Wang sequence for the mapping torus shows that the kernel of $H_{1}\left(T^{2}\right) \rightarrow H_{1}(Q)$ is generated by the class $[y]$. The homotopy exact sequence of the fibration $Q \rightarrow S^{1}$ shows that $\pi_{1}\left(T^{2}\right) \rightarrow \pi_{1}(Q)$ is injective. It then follows that the relative $\pi_{2}\left(M, T^{2}\right)$ vanishes, which implies that $\left(M, T^{2}\right)$ is weakly exact, and the homology does not inject.

Here is the plan of the paper: in the next section, we describe the general set up, including the definition of our geometric relative Seidel morphism. In Section 3, we give the proof of the main theorem up to the statement according to which the geometric Seidel morphism is an isomorphism which is proved in Section 4 of this paper. The last section gives the algebraic proof to which we referred above. In the appendix, we give the condition under which the coefficient ring can be $\mathbb{Z}$ or $\mathbb{Q}$.

Acknowledgments We are grateful to Leonid Polterovich for pointing out to us the reference [13] by Mei-Lin Yau. We would like to thank Doug Park for suggesting the Thurston manifold. We would also like to thank the referee for valuable suggestions concerning orientations.

\section{The geometric relative Seidel map}

We present the geometric Seidel morphism for Lagrangian submanifolds. In particular, we show that it is well defined and that it satisfies the properties that we need. As explained in Remark 1.2, we will work over $\mathbb{Z}_{2}$ unless explicitly specified otherwise. 


\subsection{Quantum homology of $L$}

We recall the definition of the linear cluster complex (or pearl complex) as described in Oh [11], Biran-Cornea [4] and Cornea-Lalonde [5]. Let $(M, \omega)$ be a symplectic manifold and $L \subset M$ a weakly exact Lagrangian submanifold. Let $J$ be a $\omega$-compatible almost complex structure on $M$. Then the fact that $L$ is weakly exact implies that there are no non-trivial $J$-holomorphic spheres in $M$ as well as non-trivial $J$-holomorphic discs with boundary on $L$. It follows that the quantum homology of $L$ (see [4]) is well defined and is isomorphic to $H_{*}(L) \otimes \Lambda_{L}$.

In this section, we will work in a more general setting and will only assume that $L$ is monotone, that is, there is a non-negative constant $\lambda$ such that

$$
I_{\omega}=\lambda I_{\mu}
$$

where $I_{\mu}: \pi_{2}(M, L) \rightarrow \mathbb{R}: \beta \mapsto \mu(\beta)$ is the Maslov index of $\beta$.

Suppose that $(M, L)$ is monotone with minimal Maslov index at least 2. Let $f \in$ $C^{\infty}(L)$ be a Morse function and $\rho$ a Riemannian metric on $L$ so that the pair $(f, \rho)$ be Morse-Smale. Consider the configurations of $J$-holomorphic discs connected by the negative flow lines of $f$. More precisely, let $p_{0}=x, q_{k}=y \in \operatorname{Crit}(f)$, and $u_{i}:\left(D^{2}, S^{1} ;-1,1\right) \rightarrow\left(M, L ; q_{i-1}, p_{i}\right), i=1, \ldots, k$ be $J$-holomorphic discs with boundary on $L$, such that for each pair $\left(p_{i}, q_{i}\right)$, there is an open interval $I_{i}=\left(a_{i}, b_{i}\right) \subset$ $\mathbb{R}$ and $l_{i}: I_{i} \rightarrow L$ such that

$$
\frac{d}{d t} l_{i}(t)=-\left(\nabla_{\rho} f\right)\left(l_{i}(t)\right) \quad \text { and } \quad \lim _{t \rightarrow a_{i}} l_{i}(t)=p_{i}, \quad \lim _{t \rightarrow b_{i}} l_{i}(t)=q_{i}
$$

The value $d_{i}:=b_{i}-a_{i}$ is said to be the distance between $p_{i}$ and $q_{i}$, which are $\infty$ for $i=0, k$. Let $\beta_{i}=\left[u_{i}\right] \in \pi_{2}(M, L)$ denote the class represented by the disc $u_{i}$, and set

$$
\beta:=\left(\beta_{1}, \ldots, \beta_{k}\right) \quad \text { and } \quad|\beta|:=\sum_{i=1}^{k} \beta_{i}
$$

Let $\widetilde{\mathcal{M}}(M, L ; \beta ; f, \rho, J ; x, y)$ denote the space of such configurations. We note that for each $l_{i}$ and $u_{i}$ there is a one-parameter family of reparametrization symmetries. The unparametrized moduli space is defined to be the quotient by all such symmetries:

$$
\mathcal{M}(M, L ; \beta ; f, \rho, J ; x, y):=\widetilde{\mathcal{M}}(M, L ; \beta ; f, \rho, J ; x, y) / \mathbb{R}^{2 k+1}
$$

In order for the theory to be well defined, we need regularity and transversality assumptions, as well as assumptions so that no branching of the linear cluster is possible at dimensions $\leqslant 1$ (see [4]). It is well known that all of these assumptions are satisfied in the monotone case with minimal Maslov index at least 2, and therefore in the weakly 
exact case. With all such assumptions in place, we write down the dimension of the moduli space that we have just defined. Let $\mu_{L}$ denote the Maslov class for $L$ and $|x|$ the Morse index of $x \in \operatorname{Crit}(f)$, then

$$
\operatorname{dim}_{\mathbb{R}} \mathcal{M}(M, L ; \beta ; f, \rho, J ; x, y)=|x|-|y|+\mu_{L}(|\beta|)-1
$$

The moduli spaces above are not necessarily compact as they admit real codimension 1 boundaries of three types:

(1) breaking of a Morse flow line, that is, $d_{i} \rightarrow \infty$

(2) bubbling off of a holomorphic disc, that is, $\beta_{i} \rightarrow \beta_{i}^{\prime}+\beta_{i}^{\prime \prime}$

(3) shortening of a Morse flow line, that is, $d_{i} \rightarrow 0$

Since our assumptions exclude branching of the cluster in dimension $\leqslant 1$, the bubbling off of a holomorphic disc in a linear cluster with $k$ holomorphic discs gives rise to a linear cluster with $k+1$ holomorphic discs with some $d_{i}=0$. Now consider the following union of equidimensional moduli spaces (in low dimensions)

$$
\mathcal{M}(M, L ; B ; f, \rho, J ; x, y):=\cup_{|\beta|=B} \mathcal{M}(M, L ; \beta ; f, \rho, J ; x, y)
$$

Then the type (2) and (3) boundaries cancel each other and only the type (1) boundary remains. This provides the essential idea of the following proposition (see Oh [11], Cornea-Lalonde [5] and Biran-Cornea [4]).

Proposition 2.1 The linear cluster complex (or pearl complex) of $(M, L)$ is given by the following differential $\partial_{\text {Pearl }}$ on $\operatorname{Crit}(f) \otimes \Lambda_{L}$,

$$
\partial_{\text {Pearl }} x=\sum_{y, B} \# \mathcal{M}(M, L ; B ; f, \rho, J ; x, y) e^{B} y
$$

where $\Lambda_{L}$ is the Novikov ring for $L$, and where the counting is performed for $0-$ dimensional moduli spaces only. The differential satifies $\partial_{\text {Pearl }}^{2}=0$. The quantum homology of $L$ (in $M$ ) is defined as

$$
Q H_{*}(M, L ; f, g, J):=H_{*}\left(\operatorname{Crit}(f) \otimes \Lambda_{L}, \partial_{\text {Pearl }}\right)
$$

The coefficients $\# \mathcal{M}(M, L ; B ; f, \rho, J ; x, y)$ is the counting in $\mathbb{Z}_{2}$ or $\mathbb{Q}$. One can always work over $\mathbb{Z}_{2}$, while when $L$ is relatively spin and a relative spin structure is chosen, $\mathbb{Q}$-coefficients can be used.

We note that the differential can be written as the sum

$$
\partial_{\text {Pearl }}=\partial_{\text {Morse }}+\partial_{\text {Pearl }}^{\prime}
$$


where $\partial_{\text {Morse }}$ is the classical Morse differential and we may consider the cluster complex as a deformation of the classical Morse complex. It is shown in Cornea-Lalonde [5] and Biran-Cornea [4] that the linear cluster complex is well defined and independent of the choices made. It is therefore an invariant of $(M, L)$. We write $Q H_{*}(M, L)$ for its homology. By a PSS type argument, it is shown in [4] that $Q H_{*}(M, L)$ is isomorphic to $F H_{*}(M, L)$, the Floer homology of connecting Hamiltonian paths, whose definition in our setting is recalled in Section 4 for the convenience of the reader.

Under the weakly exact assumption, we note that $\partial_{\text {Pearl }}^{\prime}=0$ since there is no pseudoholomorphic discs representing non-trivial class. Thus, the pearl complex of $(M, L)$ is the Morse complex of $L$ with coefficients in $\Lambda_{L}$.

\subsection{Bundle over a disc}

Let $\operatorname{Ham}_{L}(M, \omega)$ be the subgroup of Hamiltonian diffeomorphisms that preserve $L$. Let $\mathcal{P}_{L} \operatorname{Ham}(M, \omega)$ consist of paths $g_{t \in[0,1]}$ in $\operatorname{Ham}(M, \omega)$ such that

$$
g_{0}=\mathrm{id} \text { and } g_{1} \in \operatorname{Ham}_{L}(M, \omega)
$$

We note that $\mathcal{P}_{L} \operatorname{Ham}(M, \omega)$ is a group where

$$
\left(g g^{\prime}\right)_{t}:=g_{t} \circ g_{t}^{\prime}
$$

Such $g=g_{t \in[0,1]}$ 's define a Hamiltonian fibration over $D^{2}$ as follows (a similar fibration was actually carried over in a different context in Akveld-Salamon [1]) .

We consider the (closed) disc with diameter 1, parametrized as

$$
D^{2}:=\left\{z \in \mathbb{C}:\left|z-\frac{i}{2}\right| \leqslant \frac{1}{2}\right\}
$$

and the half discs $D_{ \pm}^{2}=\left\{z \in D^{2} \mid \pm \Re z \geqslant 0\right\}$. Then the fibration defined by $g$ is

$$
P_{g}=D_{+}^{2} \times M \sqcup D_{-}^{2} \times M / \sim:(i t, x) \sim\left(i t, g_{t}(x)\right) \text { for } t \in[0,1]
$$

Let $\pi: P_{g} \rightarrow D^{2}$ denote the projection. On this Hamiltonian bundle, let $\tau$ be the coupling form constructed from a Hamiltonian function $K$ generating $g$, then

$$
\omega_{g}:=\tau+\kappa \pi^{*} \omega_{0}
$$

is a symplectic form on $P_{g}$. We note that along the $S^{1}$-boundary, we have the restricted bundle

$$
N:=\sqcup_{t \in S^{1}} L_{t}
$$


that is obtained as the union of the copies of $L$ in each fiber; it is a Lagrangian submanifold of $P$. Because $L$ is weakly exact, the comparison theorem in BiranCornea [4] implies that $Q H_{*}(M, L)$ is isomorphic to $F H_{*}(M, L)$. Because the fibration is Hamiltonian, it is easy to see that $\left(P_{g}, N\right)$ admits sections over $\left(D^{2}, S^{1}\right)$. A class $B \in \pi_{2}\left(P_{g}, N\right)$ is a section class if $\pi_{*}(B) \in \pi_{2}\left(D^{2}, S^{1}\right)$ is the positive generator, with respect to the natural orientation on $D^{2}$. We say that $B$ is a fiber class if $B$ is in the image of the map $\pi_{2}(M, L) \rightarrow \pi_{2}\left(P_{g}, N\right)$ induced from inclusion of the fiber.

Lemma 2.2 The following sequence of homotopy groups is exact at the middle term:

$$
\pi_{2}(M, L) \stackrel{i}{\rightarrow} \pi_{2}\left(P_{g}, N\right) \stackrel{j}{\longrightarrow} \pi_{2}\left(D^{2}, S^{1}\right)
$$

Proof It follows from the diagram chasing:

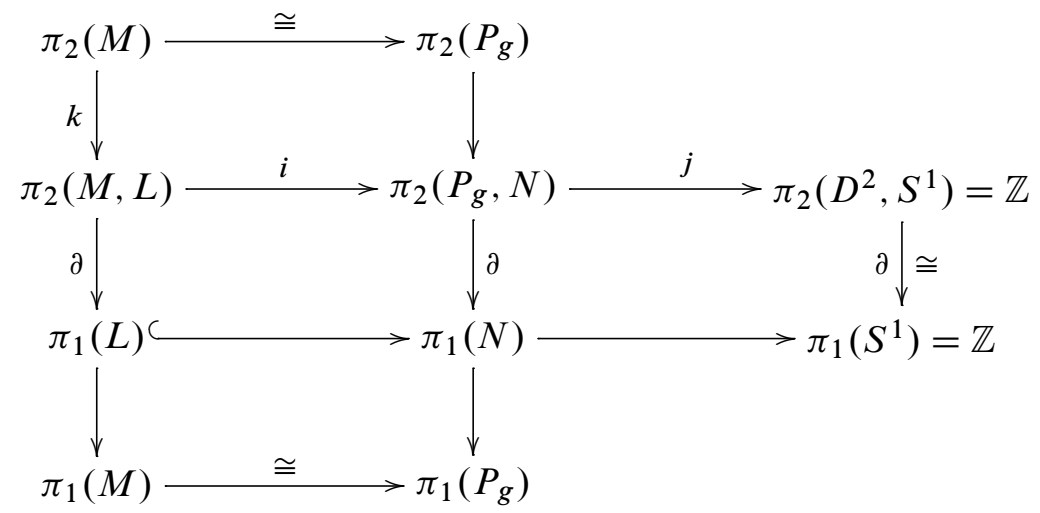

where the columns are homotopy exact sequences of pairs and all rows except the second are homotopy exact sequences of fibrations.

We only show that ker $j \subset \operatorname{img} i$. We will use $i$ and $j$ to denote the first and second map in each row. Let $\beta \in \pi_{2}\left(P_{g}, N\right)$ and $j(\beta)=0$. Then $j(\partial \beta)=0$ and by exactness of the third row, we see that there exists $\alpha^{\prime} \in \pi_{1}(L)$ such that $i\left(\alpha^{\prime}\right)=\partial \beta$. By the exactness of the columns, there is $\alpha \in \pi_{2}(M, L)$ such that $\alpha^{\prime}=\partial \alpha$ and $\partial(\beta-i(\alpha))=0$. Thus there is $\gamma \in \pi_{2}(M) \cong \pi_{2}\left(P_{g}\right)$ such that $i \circ k(\gamma)=\beta-i(\alpha)$. It follows that $\beta \in \operatorname{img} i$.

It means in particular that the difference of section classes is a fiber class.

Definition 2.3 Let the smooth map $u: D^{2} \rightarrow P_{g}$ represent $B \in \pi_{2}\left(P_{g}, N\right)$. The vertical Maslov index of $B$, denoted $\mu^{v}(B)$ is the Maslov index of the bundle pair 
$\left(u^{*} T^{v} P_{g}, u^{*} T^{v} N\right)$, where $T^{v}=\operatorname{ker} d \pi$ denotes the respective vertical tangent bundles.

One can show that the above is well defined and not dependent on the choice of $u$ (see, for example, $\mathrm{Hu}-$ Lalonde [8]). Furthermore, let $B$ and $B^{\prime}$ denote two section classes, we have

and

$$
\mu^{v}\left(B-B^{\prime}\right)=\mu_{L}\left(B-B^{\prime}\right)
$$

$$
\mu^{v}(B)=\mu_{N}(B)-2 \text {. }
$$

We can introduce the following equivalence relation among the section classes

$$
B \sim B^{\prime} \Longleftrightarrow \int_{B-B^{\prime}} \tau=0 \quad \text { and } \quad \mu^{v}\left(B-B^{\prime}\right)=\mu_{L}\left(B-B^{\prime}\right)=0
$$

An alternative construction of the fibration $P_{g}$ is the following. Let $Q:=D^{2} \times M$ and note that $N$ coincides with the mapping cylinder of $\left.g_{1}^{-1}\right|_{L}$, that is,

$$
N \cong[0,1] \times L /(0, p) \simeq\left(1, g_{1}^{-1}(p)\right) .
$$

We then have the inclusion $\rho_{g}: N \hookrightarrow Q$ given by

$$
(t, p) \mapsto\left(\theta_{t}, g_{t}^{-1}(p)\right),
$$

where $\theta_{t}:=\frac{i}{2}\left(e^{2 \pi i t}+1\right)$ in the parametrization of $S^{1}=\partial D^{2}$ by $t \in[0,1] \mapsto \theta_{t}$.

Lemma $2.4(Q, N) \cong\left(P_{g}, N\right)$ with the respective inclusion of $N$.

Proof Recall that $g \in \mathcal{P}_{L} \operatorname{Ham}(M, \omega)$ is a path starting from id:

$$
g_{0}=\mathrm{id}, g_{t \in[0,1]} \in \operatorname{Ham}(M, \omega) \quad \text { and } \quad g_{1} \in \operatorname{Ham}_{L}(M, \omega)
$$

We note that $\left(P_{g}, N\right)$ depends only on the homotopy class of $g$ with fixed end points. First we show that $(Q, N)$ depends only on such homotopy class as well. Let $g^{\prime} \sim g$ be homotopic to $g$ in $\mathcal{P}_{L} \operatorname{Ham}(M, \omega)$ with fixed end points. Thus

$$
h_{t}=g_{t}^{\prime} \circ g_{t}^{-1} \in \Omega \operatorname{Ham}(M, \omega) \text { is a contractible loop in } \operatorname{Ham}(M, \omega)
$$

Let $\Psi^{h}: D^{2} \rightarrow \operatorname{Ham}(M, \omega)$ be a homotopy of $\left\{h_{t}\right\}$ to id $\in \operatorname{Ham}(M, \omega)$, where $\Psi^{h}\left(\theta_{t}\right)=h_{t}$ and $\Psi^{h}\left(\frac{i}{2}\right)=\mathrm{id}$. Then we have

$$
\Psi: Q \stackrel{\cong}{\rightarrow} Q:(z, x) \mapsto\left(z, \Psi_{z}^{h}(x)\right), \quad \text { and thus } \rho_{g^{\prime}}=\Psi^{h} \circ \rho_{g}: N \hookrightarrow Q .
$$


We describe an alternative construction of $P_{g}$, together with the embedding of $N$. Consider the map $\psi: D_{+}^{2} \times M \rightarrow D_{+}^{2} \times M$ given by

$$
\psi(z, x)=\left(z, g_{t}^{-1}(x)\right)
$$

where $z=s+i t \in D_{+}^{2}$. We define $Q$ from the quotient

$$
D_{+}^{2} \times M \sqcup D_{-}^{2} \times M / \sim^{\prime}:(t, x) \sim^{\prime}(t, x) \text { for } t \in[0,1]
$$

Then the map

$$
D_{+}^{2} \times M \sqcup D_{-}^{2} \times M \stackrel{\psi \sqcup \text { نid }}{\longrightarrow} D_{+}^{2} \times M \sqcup D_{-}^{2} \times M
$$

induces an isomorphism of $Q \stackrel{\cong}{\rightarrow} P_{g}$. The inclusions of $N$ obviously correspond.

\subsection{Definition of the Seidel map}

We first recall the definition of the map $i_{*}: H_{*}(L) \rightarrow H_{*}(N)$ via Morse homology. Denoting the basis of the fibration $N$ as the set of points in the unit circle of the complex plane and by $L_{ \pm 1}$ the two fibers over +1 and -1 , let $F \in C^{\infty}(N)$ satisfy the following:

- $F$ is a Morse function

- $f_{ \pm}:=\left.F\right|_{L_{ \pm 1}}$ are Morse functions on $L$

- $\operatorname{Crit}(F)=\operatorname{Crit}\left(f_{+}\right) \cup \operatorname{Crit}\left(f_{-}\right)$and

- $\max f_{-}+1<\min f_{+}$.

We choose a metric $G$ on $N$ such that the pairs $(F, G)$ and $\left(f_{ \pm}, g_{ \pm}\right)$are Morse-Smale pairs on $N$ and $L$ respectively, where $g_{ \pm}$are the restrictions of $G$ to $L_{ \pm 1}$. Then the Morse complexes are well defined and compute the homologies of the respective manifolds. To define $i_{*}$ via Morse theory, we require that the pair $(F, G)$ satisfies the following:

- In a neighbourhood of the fibers over \pm 1 , the fibration $N \rightarrow S^{1}$ is locally identified as a product, $L \times U_{ \pm}$, where $\pm 1 \in U_{ \pm} \subset S^{1}$.

- The restriction of $F$ to this neighbourhood is of the form $f_{ \pm}+\varphi_{ \pm}$, where $\varphi_{ \pm}: U_{ \pm} \rightarrow \mathbb{R}$ is smooth with unique critical point at \pm 1 .

- The restriction of $G$ to this neighbourhood is a product metric. 
It follows that the map induced by the inclusion of the set of critical points is an inclusion of Morse complexes:

$$
i: M C_{*}\left(L ; f_{-}, g_{-}\right) \hookrightarrow M C_{*}(N ; F, G)
$$

It induces the map $i_{*}: H_{*}(L) \rightarrow H_{*}(N)$ in homology.

We now define the Seidel map in this setting. Suppose that $J$ is a tamed almost complex structure on $P_{g}$, where we may choose the symplectic structure on $P_{g}$ to be the pull-back of the product symplectic structure on $Q$ via the isomorphism given by Lemma 2.4. We also suppose that $J$ is compatible with the fibration, namely:

- the projection $\pi: P_{g} \rightarrow D^{2}$ is pseudo-holomorphic, and

- $J$ restricts to compatible almost complex structures on the fibers.

We consider the linear clusters in $\left(P_{g}, N\right)$ for which exactly one of the pseudoholomorphic discs represents a section class in $\pi_{2}\left(P_{g}, N\right)$ and all other discs represent fiber classes. Given $x_{-} \in \operatorname{Crit}\left(f_{-}\right)$and $y_{+} \in \operatorname{Crit}\left(f_{+}\right)$, this amounts to consider the moduli spaces $\mathcal{M}\left(P_{g}, N ; \sigma ; F, G, J ; x_{-}, y_{+}\right)$where $|\sigma|$ is a section class. Let $|\cdot|^{L}$ be the Morse index in $L$ and $|\cdot|^{N}$ that in $N$, then

$$
\left|x_{-}\right|^{N}=\left|x_{-}\right|^{L} \text { and }\left|y_{+}\right|^{N}=\left|y_{+}\right|^{L}+1
$$

We obtain from (2-1)

$$
\begin{aligned}
\operatorname{dim}_{\mathbb{R}} \mathcal{M}\left(P_{g}, N ; \sigma ; F, G, J ; x_{-}, y_{+}\right) & =\left|x_{-}\right|^{N}-\left|y_{+}\right|^{N}+\mu_{N}(|\sigma|)-1 \\
& =\left|x_{-}\right|^{L}-\left|y_{+}\right|^{L}+\mu^{v}(|\sigma|)
\end{aligned}
$$

Definition 2.5 Let $\sigma$ denote a section class in $\pi_{2}(P, N)$ and $\sigma_{0}$ a particular choice of reference section class. Then $B:=\sigma-\sigma_{0}$ is a fiber class and the chain level geometric Seidel map is

$$
\begin{aligned}
& \Psi_{L}\left(g, \sigma_{0}\right): \operatorname{Crit}\left(f_{-}\right) \otimes \Lambda_{L-} \rightarrow \operatorname{Crit}\left(f_{+}\right) \otimes \Lambda_{L_{+}} \\
& \Psi_{L}\left(g, \sigma_{0}\right)\left(x_{-}\right):=\sum_{B, y_{+}} \# \mathcal{M}\left(P_{g}, N ; \sigma_{0}+B ; F, G, J_{P} ; x_{-}, y_{+}\right) e^{B} y_{+}
\end{aligned}
$$

where the coefficients count (in $\mathbb{Z}$ or $\mathbb{Z}_{2}$, see Remark 2.6) the zero dimensional moduli spaces.

Remark 2.6 We note that for the purpose of the main theorem, with the assumption of weak exactness on $L$, standard transversality provides that the moduli spaces above are smooth manifolds. In general, when we allow $(M, L)$ to be monotone with minimal Maslov index 2, the transversality arguments in Biran-Cornea [4] can be adapted so 
that the moduli spaces are again smooth with the expected dimension in dimension $\leqslant 1$. The main point in the adaptation is that the disc that represents a section class is necessarily simple.

For the counting, one can always work over $\mathbb{Z}_{2}$. On the other hand, if $L$ is relatively spin and the map $g_{1}$ preserves the chosen relative spin structure, we may use $\mathbb{Z}$ or $\mathbb{Q}$ as coefficients, see Section A, the appendix.

Lemma 2.7 $\Psi_{L}\left(g, \sigma_{0}\right)$ is a chain map of degree $\mu^{v}\left(\sigma_{0}\right)$ and the induced map on $Q H_{*}(M, L)$, does not depend on the choice of generic data.

Proof Because $(M, L)$ is weakly exact, the moduli spaces of holomorphic discs in $P_{g}$ with boundary in $N$, representing a section class, are compact. Let $B$ denote a fiber class in $\pi_{2}\left(P_{g}, N\right)$. Thus, $\mathcal{M}\left(P_{g}, N ; \sigma_{0}+B ; F, G, J_{P} ; x_{-}, y_{+}\right)$is compactified by broken Morse flow lines. From this, it follows that $\Psi_{L}\left(g, \sigma_{0}\right)$ is a chain map. Because $\operatorname{deg} e^{B}=-\mu_{L}(B)$, by the dimension computation of the moduli spaces, we see that the degree of the map is $\mu^{v}\left(\sigma_{0}\right)$.

Now we show that it does not depend on the choices of generic data $\left(F, G, J_{P}\right)$. Suppose that we have two triples of Morse functions, metrics and compatible almost complex structures: $\left(F_{i}, G_{i}, J_{P, i}\right)$ for $i=0,1$. Consider the fibration $(\tilde{P}, \tilde{N})=$ $\left(P_{g}, N\right) \times[0,1]$ and endow it with a triple $(\widetilde{F}, \widetilde{G}, \widetilde{J})$ where $\widetilde{F}$ is a smooth Morse function on $\tilde{N}, \widetilde{G}$ is a metric on $\tilde{N}$ that restricts to $G_{i}$ on $P_{g} \times\{i\}$ and where $\widetilde{J}$ is a smooth family of $\omega$-compatible and fibration-compatible complex structures on $P_{g} \times\{t\}$, which connects $J_{P, i}$ on $P_{g} \times\{i\}$. Assume that $\tilde{F}$ coincides with $\widetilde{F}_{i}:=A_{i} F_{i}+C_{i}$ on $\left(P_{g}, N\right) \times\{i\}$ for some constants $A_{i}$ and $C_{i}, i=0,1$ chosen so that

$$
\min f_{0,+}>\max f_{1,+}+1>\min f_{1,+}>\max f_{0,-}+1>\min f_{0,-}>\max f_{1,-}+1
$$

Here $f_{i, \pm}$ is the restriction of $\widetilde{F}$ to the fiber $L_{i, \pm}$ of $N \times[0,1] \rightarrow S^{1} \times[0,1]$ above the point $( \pm 1, i)$. Assume moreover as usual that $\operatorname{Crit}(\widetilde{F})=\operatorname{Crit}\left(\widetilde{F}_{0}\right) \cup \operatorname{Crit}\left(\widetilde{F}_{1}\right)$.

In general, we consider the configuration of a linear cluster in $\widetilde{P}$ connecting $x_{-} \in L_{0,-}$ to $y_{+} \in L_{1,+}$ via negative gradient lines and holomorphic discs $u_{i}:\left(D^{2}, S^{1}\right) \rightarrow$ $\left(P_{g}, N\right) \times\left\{t_{i}\right\}$ for some $t_{i} \in[0,1]$, the total class of the $\left[u_{i}\right]$ 's being a section class $\sigma \in \pi_{2}(\tilde{P}, \tilde{N}) \cong \pi_{2}\left(P_{g}, N\right)$. Exactly like in the definition of $\Psi_{L}$, we can adapt the transversality argument in Biran-Cornea [4] and see that the moduli spaces of such configurations are smooth of the expected dimension when the dimension is $\leqslant 1$. Let $\mathcal{M}\left(\widetilde{P}, \widetilde{N} ; \sigma ; \widetilde{F}, \widetilde{G}, \widetilde{J} ; x_{-}, y_{+}\right)$denote the moduli space of such linear clusters and 
write $\sigma=\sigma_{0}+B$, where $\sigma_{0}$ is a chosen reference section class and $B$ is a fiber class. We define $\Sigma: \operatorname{Crit}\left(\widetilde{F}_{0,-}\right) \otimes \Lambda_{L} \rightarrow \operatorname{Crit}\left(\widetilde{F}_{1,+}\right) \otimes \Lambda_{L}$ by

$$
\Sigma\left(x_{-} ; \sigma_{0}\right):=\sum_{B, y_{+}} \# \mathcal{M}\left(\widetilde{P}, \tilde{N} ; \sigma_{0}+B ; \widetilde{F}, \widetilde{G}, \widetilde{J} ; x_{-}, y_{+}\right) e^{B} y_{+}
$$

where the counting $\# \mathcal{M}$ is for the moduli spaces of expected dimension 0 , that is,

$$
\left|x_{-}\right|^{L}-\left|y_{+}\right|^{L}+\mu^{v}\left(\sigma_{0}\right)+\mu_{L}(B)+1=0
$$

It follows that $\Sigma\left(\cdot ; \sigma_{0}\right)$ is of degree $\mu^{v}\left(\sigma_{0}\right)+1$.

Now considering the boundary components of the moduli spaces with dimension 1 , we see that it consists of the following four types of configurations, corresponding precisely to the case when the breaking of Morse flow lines happens on one of the $L_{i, \pm}$ 's.

It is then obvious that:

(1) the breaking on $L_{0,-}$ corresponds to $\Sigma\left(\cdot ; \sigma_{0}\right) \circ \partial_{\text {Pearl }}$,

(2) the breaking on $L_{1,+}$ corresponds to $\partial_{\text {Pearl }} \circ \Sigma\left(\cdot ; \sigma_{0}\right)$,

(3) the breaking on $L_{0,+}$ corresponds to $\Phi_{+} \circ \Psi_{L_{0}}\left(g, \sigma_{0}\right)$ and

(4) the breaking on $L_{1,-}$ corresponds to $\Psi_{L_{1}}\left(g, \sigma_{0}\right) \circ \Phi_{-}$,

where

$$
\Phi_{ \pm}: \operatorname{Crit}\left(f_{0, \pm}\right) \otimes \Lambda_{L} \rightarrow \operatorname{Crit}\left(f_{1, \pm}\right) \otimes \Lambda_{L}
$$

are the comparison maps between quantum homologies of $L$ with different choices of $(f, \rho, J)$, which are quasi-isomorphisms. We can then write down

$$
\Sigma\left(\cdot ; \sigma_{0}\right) \circ \partial_{\text {Pearl }}-\partial_{\text {Pearl }} \circ \Sigma\left(\cdot ; \sigma_{0}\right)=\Phi_{+} \circ \Psi_{L_{0}}\left(g, \sigma_{0}\right)-\Psi_{L_{1}}\left(g, \sigma_{0}\right) \circ \Phi_{-}
$$

and it follows that $\Phi_{+} \circ \Psi_{L_{0}}\left(g, \sigma_{0}\right)$ and $\Psi_{L_{1}}\left(g, \sigma_{0}\right) \circ \Phi_{-}$induce the same maps on quantum homologies.

We note that the above lemma implies that the Seidel map $\Psi\left(g, \sigma_{0}\right)$ does not depend on the homotopy class of $g$, because the construction for $g^{\prime} \sim g$ gives the same bundles $\left(P_{g}, N\right)$ with a different set of data $\left(F, G, J_{P}\right)$. 


\section{Proof of the theorem}

We will use the following proposition, which follows from Proposition 4.1 in Section 4:

Proposition 3.1 The map $\Psi_{L}\left(g, \sigma_{0}\right)$ is an isomorphism of quantum homology of $(M, L)$.

As shown in the introduction, the main theorem (Theorem 1.1) follows from:

Lemma 3.2 Let $(M, L)$ be symplectically aspherical. Then $i_{*}: H_{*}(L) \rightarrow H_{*}(N)$ is injective.

Proof Note that the Morse theoretical definition of $i_{*}$ is defined by the inclusion of chain complexes $M C_{*}(L) \hookrightarrow M C_{*}(N)$. Since $(M, L)$ is symplectically aspherical, $Q H_{*}(M, L)=H_{*}\left(L ; \Lambda_{L}\right)$ is the homology of $\left(M C_{*}(L) \otimes \Lambda_{L}, \partial_{\text {Morse }}\right)$. The Seidel map $\Psi_{L}\left(g, \sigma_{0}\right)$ then defines an isomorphism of $Q H_{*}(M, L)$.

By the universal coefficient theorem, $H_{*}(L) \otimes \Lambda_{L}$ is a subgroup of $Q H_{*}(M, L)$. We prove by contradiction. Suppose that $\operatorname{ker} i_{*} \neq\{0\}$, then there exists $\alpha \neq 0 \in \operatorname{ker} i_{*}$ such that it is represented by $\sum_{i} a_{i} x_{i,-} \in M C_{*}\left(L_{-}\right)$and

$$
\sum_{i} a_{i} x_{i,-}=\partial_{\text {Morse }}^{N} \sum_{j} b_{j} y_{j,+} \text { for some } y_{j,+} \in \operatorname{Crit}\left(f_{+}\right)
$$

where $\partial_{\text {Morse }}^{N}$ denote the boundary operator in Morse homology of $N$. Since $\Psi_{L}$ is an isomorphism on $Q H_{*}(M, L)$, we have $\Psi_{L}\left(\sum_{i} a_{i} x_{i,-}\right) \neq 0$.

We work on chain level. Let $y \in \operatorname{Crit}(F)$ and $z_{+} \in \operatorname{Crit}\left(f_{+}\right) \subset \operatorname{Crit}(F)$ and consider the moduli space for fiber classes $B$ :

$$
\mathcal{M}\left(P_{g}, N ; \sigma_{0}+B ; F, G, J_{P} ; y, z_{+}\right),
$$

which has expected dimension

$$
|y|^{N}-\left|z_{+}\right|^{N}+\mu_{N}\left(\sigma_{0}+B\right)-1=|y|^{N}-\left|z_{+}\right|^{L}+\mu^{v}\left(\sigma_{0}\right)+\mu_{L}(B) .
$$

Let $\Sigma\left(g, \sigma_{0}\right)$ denote the map $M C_{*}(N) \mapsto M C_{*}(L) \otimes \Lambda_{L}$ defined by

$$
y \mapsto \sum_{B, z_{+}} \# \mathcal{M}\left(P_{g}, N ; \sigma_{0}+B ; F, G, J_{P} ; y, z_{+}\right) e^{B} z_{+}
$$

where the coefficients count dimension 0 moduli spaces. We note that when restricted to $M C_{*}\left(L_{-}\right), \Sigma\left(g, \sigma_{0}\right)$ coincides with $\Psi_{L}$. 
Consider now the moduli spaces $\mathcal{M}\left(P_{g}, N ; \sigma_{0}+B ; F, G, J_{P} ; y, z_{+}\right)$of dimension 1 , which are compactified by the breakings in the Morse flow lines in $N$, on $L_{ \pm}$. By the choices made for $(F, G)$, we write down the boundary components for $y=y_{+}$:

$$
\begin{aligned}
& \mathcal{M}_{\text {Morse }}\left(y_{+}, y_{+}^{\prime}\right) \times \mathcal{M}\left(P_{g}, N ; \sigma_{0}+B ; F, G, J_{P} ; y_{+}^{\prime}, z_{+}\right) \\
& \mathcal{M}_{\text {Morse }}\left(y_{+}, x_{-}\right) \times \mathcal{M}\left(P_{g}, N ; \sigma_{0}+B ; F, G, J_{P} ; x_{-}, z_{+}\right) \\
& \mathcal{M}_{\text {Morse }}\left(z_{+}^{\prime}, z_{+}\right) \times \mathcal{M}\left(P_{g}, N ; \sigma_{0}+B ; F, G, J_{P} ; y_{+}, z_{+}^{\prime}\right)
\end{aligned}
$$

where $\mathcal{M}_{\text {Morse }}$ denotes the moduli space of Morse trajectories (in $N$ ) connecting the two critical points, which in this case, all have dimension 0 . It follows that

$$
\Sigma\left(g, \sigma_{0}\right) \circ \partial_{\text {Morse }}^{N}\left(y_{+}\right)+\partial_{\text {Morse }} \circ \Sigma\left(g, \sigma_{0}\right)\left(y_{+}\right)=0
$$

Thus

$$
\begin{aligned}
\Psi_{L}\left(\sum_{i} a_{i} x_{i,-}\right) & =\Sigma\left(g, \sigma_{0}\right)\left(\sum_{i} a_{i} x_{i,-}\right) \\
& =\sum_{j} b_{j} \Sigma\left(g, \sigma_{0}\right) \circ \partial_{\text {Morse }}^{N}\left(y_{j,+}\right) \\
& =\partial_{\text {Morse }}\left(-\sum_{j} b_{j} \Sigma\left(g, \sigma_{0}\right)\left(y_{j,+}\right)\right)
\end{aligned}
$$

and becomes 0 in $Q H_{*}(M, L)$. This is a contradiction.

\section{Correspondence between the analytic and geometric Seidel maps}

We first recall the construction of the analytic Seidel map and restate it in the current geometric setting. Then we show that the two constructions coincide. In a way similar to Seidel [12], the comparison uses the PSS isomorphism in the Lagrangian setting, for which we will adapt the construction of Biran-Cornea [4] or Hu-Lalonde [8] using Hamiltonian fibrations.

\subsection{Analytic setting}

In this subsection, we recall the Seidel map and PSS isomorphism in the analytic setting. Recall that

$$
D_{ \pm}^{2}=\left\{z \in \mathbb{C}:\left|z-\frac{i}{2}\right| \leqslant \frac{1}{2} \text { and } \pm \Re z \geqslant 0\right\}
$$

Let $\partial_{0}=D_{+}^{2} \cap i \mathbb{R}=D_{-}^{2} \cap i \mathbb{R}$, and $\partial_{ \pm}=D_{ \pm}^{2} \cap\left\{z:\left|z-\frac{i}{2}\right|=\frac{1}{2}\right\}$. Let $\mathcal{P}_{L} M$ be the space of paths in $M$ with both ends on $L$ that are contractible in $(M, L)$, and $\widetilde{\mathcal{P}}_{L} M$ 
the covering space whose elements are equivalence classes $[l, w]$ of pairs $(l, w)$

$$
l:([0,1],\{0,1\}) \rightarrow(M, L) \text { and } w:\left(D_{+}^{2}, \partial_{+}, \partial_{0}\right) \rightarrow(M, L, l)
$$

where $l(t)=w(i(1-t))$. The equivalence relation is the following

$$
(l, w) \sim\left(l^{\prime}, w^{\prime}\right) \Longleftrightarrow l=l^{\prime} \text { and } w \sim \partial_{0} w^{\prime}
$$

where $\sim_{\partial_{0}}$ means that the homotopy connecting $w$ and $w^{\prime}$ in $(M, L)$ becomes the trivial homotopy when restricted to $\partial_{0}$. The Floer homology of $(M, L)$ is constructed from a choice of a time-dependent Hamiltonian function $H$ and a compatible almost complex structure $J$ on $M$. The action functional as well as the metric are then defined as:

$$
a_{H}([l, w])=-\int_{D_{+}^{2}} w^{*} \omega+\int_{[0,1]} H_{t}(l(t)) d t
$$

and

$$
(\xi, \eta)_{J}=\int_{[0,1]} \omega\left(\xi(t), J_{t} \eta(t)\right) d t, \text { for } \xi, \eta \in C^{\infty}\left(l^{*} T M\right)
$$

Then the Floer homology $F H_{*}(M, L ; H, J)$ can be seen as the Morse homology of $\widetilde{P}_{L} M$ for $a_{H}$ in the metric $(,)_{J}$. The differential $d a_{H}$ and $(,)_{J}$ are well defined on $P_{L} M$ already and the equation of negative gradient flow lines can be written in $P_{L} M$ as well:

$$
\begin{cases}\frac{\partial u}{\partial s}+J_{t}(u)\left(\frac{\partial u}{\partial t}-X_{H_{t}}(u)\right)=0 & \text { for all }(s, t) \in \mathbb{R} \times[0,1], \\ u: \mathbb{R} \times[0,1] \rightarrow M & \text { and }\left.u\right|_{\mathbb{R} \times\{0,1\} \subset L} \subset L\end{cases}
$$

The action of $\mathcal{P}_{L} \operatorname{Ham}(M, \omega)$ on $\mathcal{P}_{L} M$ lifts to an action of an extension group $\tilde{\mathcal{P}}_{L} \operatorname{Ham}(M, \omega)$ on $\tilde{\mathcal{P}}_{L} M$. An element of $\widetilde{\mathcal{P}}_{L} \operatorname{Ham}(M, \omega)$ can be represented as $(g, \tilde{g})$, where $g \in \mathcal{P}_{L} \operatorname{Ham}(M, \omega)$ and $\widetilde{g}$ is determined by the action of $(g, \tilde{g})$ on point elements $[p, p] \in \widetilde{\mathcal{P}}_{L} M$ in the following fashion. Let's denote $(g, \widetilde{g}) \circ[p, p]$ by $\left[p^{g}, w_{p}^{g}\right]$. Let $[l, w] \in \widetilde{\mathcal{P}}_{L} M$ and $\left[l^{g}, w^{g}\right]=(g, \tilde{g}) \circ[l, w]$, then

$$
l^{g}(t)=g_{t} \circ l(t)
$$

and $w^{g}$ is defined as follows (we write $w^{g}$ instead of $w^{(g, \tilde{g})}$ to simplify the notation). Consider $w$ as a homotopy from a constant path $l(0)$ to $l$, that is, it is spanned by a one-parameter family $\alpha_{\tau \in\left[\frac{1}{2}, 1\right]}$ with $\alpha_{\frac{1}{2}}(t)=l(0)$ and $\alpha_{1}(t)=l(t)$. The action of $g$ on $w$ is then the strip obtained as the image $g_{t}\left(\alpha_{\tau}(t)\right)$. Now we define the action of $(g, \tilde{g})$ on $w$ as the half disk obtained by gluing the above strip along its boundary at $\tau=\frac{1}{2}$ with $w_{l(0)}^{g}$. This defines $w^{g}$.

Now the push-forward by $g$ of $H$ and $J$ is given by the pair $\left(H^{g}, J^{g}\right)$ :

$$
H^{g}(t, x)=H\left(t, g_{t}^{-1}(x)\right)+K(t, x) \quad \text { and } \quad J_{t}^{g}=d g_{t} \circ J_{t} \circ d g_{t}^{-1}
$$


where $K(t, x)$ is the Hamiltonian function generating $g$. The lifted action of $(g, \widetilde{g})$ on $\widetilde{P}_{L} M$ then defines an isomorphism of Floer homologies

$$
\Psi_{\tilde{g}}: F H_{*}(M, L ; H, J) \rightarrow F H_{*}\left(M, L ; H^{g}, J^{g}\right):[l, w] \mapsto\left[l^{g}, w^{g}\right]
$$

which gives the analytic version of the relative Seidel map.

We also describe the PSS isomorphism in the analytic setting. We will describe only the direction $\mathrm{QH}_{*} \rightarrow \mathrm{FH}_{*}$ because the other direction is similar, and we will describe (both directions of) the geometric version in more detail in the next subsection. Let $Z_{-}$be the half-disc with infinite end:

$$
Z_{-}:=D_{-}^{2} \cup_{\partial_{0}}\left(\mathbb{R}^{+} \times i[0,1]\right) \subset \mathbb{C}
$$

and choose a $C^{\infty} z$-dependent $\left(z \in Z_{-}\right.$) Hamiltonian function and a compatible almost complex structure $(\mathbf{H}, \mathbf{J}):=\left\{\left(H_{z}, J_{z}\right)\right\}_{z \in Z_{-}}$on $M$ such that

and

$$
\begin{aligned}
\left.(\mathbf{H}, \mathbf{J})\right|_{z \in D_{-}^{2}} & =\left(0, J_{0}\right) \\
\left.(\mathbf{H}, \mathbf{J})\right|_{\Re z>1} & =(H, J),
\end{aligned}
$$

where $J_{0}$ is a generic compatible almost complex structure. The equation is

$$
\begin{cases}\frac{\partial u}{\partial s}+J_{z}(u)\left(\frac{\partial u}{\partial t}-X_{H_{z}}(u)\right)=0 & \text { for all } z=s+i t \in Z_{-} \\ u: Z_{-} \rightarrow M & \text { and }\left.u\right|_{\partial Z_{-} \subset L} \subset L\end{cases}
$$

Then finite energy solutions converge to critical points $l$ of $d a_{H}$ as $s \rightarrow \infty$. We may mark the point $\frac{1}{2}(-1, i)$ and consider the evaluation map from the moduli space of the solutions to (4-2). The PSS isomorphism $Q H_{*} \rightarrow F H_{*}$ is then defined by counting the intersections with cycles in $L_{-}:=L \times\left\{\frac{1}{2}(-1, i)\right\}$ of the moduli space under the evaluation.

\subsection{Geometric setting}

In this subsection, we rewrite the construction of PSS isomorphism in the previous subsection using Hamiltonian fibrations, and prove Proposition 4.1 stating that the geometric and analytic Seidel morphisms coincide.

The equation (4-2) can be written also as the $\bar{\partial}$-equation for a holomorphic section in a fibration over $Z_{-}$as follows. Let $P_{-}=Z_{-} \times M$ and consider the symplectic form:

$$
\Omega_{-}:=\kappa\left(\omega+d H_{z} \wedge d t\right)+d s \wedge d t
$$

The almost complex structure $\widetilde{J}_{-}$on $P_{-}$is given by $J_{z}$ along the fibers and

$$
\tilde{J}_{-}\left(\frac{\partial}{\partial s}\right)=\frac{\partial}{\partial t}-X_{H_{z}}
$$


The $\left\{\left(H_{z}, J_{z}\right)\right\}_{z \in Z_{-}}$in the above expressions are given by the same $(\mathbf{H}, \mathbf{J})$ in the previous subsection. Then $\widetilde{J}_{-}$is tamed by $\Omega_{-}$and the $\bar{\partial}$-equation for $\widetilde{J}_{-}-$holomorphic sections $\sigma_{-}: Z_{-} \rightarrow P_{-}$with boundary on $L \times \partial Z_{-}$coincides with (4-2) and the graph of a solution of (4-2) gives a $\widetilde{J}_{-}$-holomorphic section. Thus we obtain the geometric version of the PSS isomorphism in the direction $Q H_{*} \rightarrow F H_{*}$.

To define the other direction, $F H_{*} \rightarrow Q H_{*}$, let $\tau: \mathbb{C} \rightarrow \mathbb{C}$ be the anti-linear map reversing the real part:

$$
\tau(s+i t)=-s+i t .
$$

then $D_{+}=\tau\left(D_{-}\right)$and we consider $Z_{+}=\tau\left(Z_{-}\right), P_{+}=Z_{+} \times M$. From $(\mathbf{H}, \mathbf{J})$ as above, we define $\left\{\left(H_{\tau(z)}^{g}, J_{\tau}^{g}(z)\right)\right\}_{z \in Z_{+}}$to be the push-forward by $g$. The corresponding symplectic structure $\Omega_{+}^{g}$ is

$$
\Omega_{+}^{g}:=\kappa\left(\omega+d H_{\tau(z)}^{g} \wedge d t\right)+d s \wedge d t
$$

and the tamed almost complex structure $\widetilde{J}_{+}^{g}$ is defined by $J_{\tau(z)}$ along the fiber over $z \in Z_{+}$and

$$
J_{+}\left(\frac{\partial}{\partial s}\right)=\frac{\partial}{\partial t}-X_{H_{\tau(z)}^{g}}
$$

In this case, pseudoholomorphic sections with finite energy converge to critical points $l^{g}$ of $d a_{H^{g}}$ as $s \rightarrow-\infty$. Then counting of moduli spaces of sections gives the other direction $\mathrm{FH}_{*} \rightarrow Q H_{*}$ of the PSS isomorphism.

Proposition 4.1 The composition

$$
Q H_{*}(M, L) \stackrel{P S S}{\longrightarrow} F H_{*}(M, L) \stackrel{\Psi_{\widetilde{g}}}{\longrightarrow} F H_{*}(M, L) \stackrel{P S S}{\longrightarrow} Q H_{*}(M, L)
$$

coincides with the geometric Seidel map $\Psi_{L}\left(g, \sigma_{0}\right)$ for an appropriately chosen $\sigma_{0}$.

Proof The proof is an application of the gluing method to explicitly identify the moduli spaces involved. Note that this only concerns the moduli spaces of dimension $\leqslant 1$. It is analogous to the proof showing that PSS maps are isomorphisms (see Biran-Cornea [4]).

Consider for each $R>0$ the map $G_{R}:[1, R+1] \times[0,1] \times M \rightarrow[-R-1,-1] \times[0,1] \times M$ between subsets of $P_{-}$and $P_{+}$given by

$$
G_{R}(s, t, x)=\left(s-R-2, t, g_{t}(x)\right)
$$

It is then straightforward to check that $G_{R *}\left(\Omega_{-}\right)=\Omega_{+}^{g}$. It follows that $P_{+}$and $P_{-}$ may be glued symplectically using $G_{R}$ :

$$
P_{g}(R):=\left\{\left(P_{-} \backslash(R+1, \infty) \times[0,1] \times M\right) \cup\left(P_{+} \backslash(-\infty,-R-1) \times[0,1] \times M\right)\right\} / G_{R},
$$


and we obtain a Hamiltonian fibration, which is homotopic to $P_{g}$ given in the previous geometric construction. The symplectic form $\Omega(R)$ on $P_{g}(R)$ can be written as

$$
\Omega(R)=\kappa \tau(R)+\omega_{D^{2}}
$$

where $\tau(R)$ is a coupling form.

The almost complex structures on $P_{+}$and $P_{-}$are glued by $G_{R}$ as well, and the result is denoted $\widetilde{J}_{g}(R)$. For pseudoholomorphic sections $u_{+}$and $u_{-}$in $P_{+}$and $P_{-}$with finite energy, which converge to $\left[l^{g}, w^{g}\right]$ and $[l, w]$ respectively, their limits as $s \rightarrow \pm \infty$ are identified naturally by $g$. It follows that under gluing by $G_{R}, u_{+}$ and $u_{-}$give rise to a pseudoholomorphic section of $P_{g}(R)$, for $R$ big enough, using standard gluing argument. Let $\mathcal{M}\left(P_{+}, l^{g}\right)$ and $\mathcal{M}\left(P_{-}, l\right)$ be the moduli spaces of pseudoholomorphic sections in $P_{+}$and $P_{-}$with prescribed limits at infinity, and $\mathcal{M}\left(P_{g}(R)\right)$ the moduli space of pseudoholomorphic sections in $P_{g}(R)$. The same gluing argument (together with compactness) shows that for all $R>0$,

$$
\mathcal{M}\left(P_{g}(R)\right) \cong \cup_{l} \mathcal{M}\left(P_{+}, l^{g}\right) \times \mathcal{M}\left(P_{-}, l\right)
$$

Next we identify the maps. Let $\alpha_{-}$denote a chain in $L_{-}$and $\tilde{l}=[l, w]$. Let $\mathcal{M}\left(P_{-}, \tilde{l}\right)$ be the subspace of $\mathcal{M}\left(P_{-}, l\right)$ such that

$$
I_{\mu}\left(w \# u_{-}\right)=0
$$

where we think of $u_{-}$as a map from the half disc to $M$ via the projection $P_{-} \rightarrow M$. Then the PSS map $Q H_{*} \rightarrow F H_{*}$ is defined at the chain level as

$$
P S S\left(\alpha_{-}\right)=\sum_{\tilde{l}} \#\left(\mathrm{ev}_{-} \mathcal{M}\left(P_{-}, \tilde{l}\right) \pitchfork \alpha_{-}\right) \tilde{l}
$$

where $e_{-}$is the evaluation map

$$
\mathcal{M}\left(P_{-}, \tilde{l}\right) \rightarrow L_{-}: u_{-} \mapsto u_{-}\left(\frac{1}{2}(-1, i)\right)
$$

On the other hand, let $\alpha_{+}$be a chain in $L_{+}=L \times\left\{\frac{1}{2}(1, i)\right\}$ and $\mathcal{M}\left(P_{+}, \tilde{l}^{g}\right)$ be the subspace of $\mathcal{M}\left(P_{+}, l^{g}\right)$ such that

$$
I_{\mu}\left(w^{g} \#\left(-u_{+}\right)\right)=0 \text { for } u_{+} \in \mathcal{M}\left(P_{+}, \tilde{l}^{g}\right)
$$

Then the PSS map $\mathrm{FH}_{*} \rightarrow Q H_{*}$ is defined as

$$
\operatorname{PSS}\left(\tilde{l}^{g}\right)=\sum_{B \in \pi_{2}(M, L), \alpha_{+}} \#\left(\mathrm{ev}_{+} \mathcal{M}\left(P_{+}, \tilde{l}^{g} \# B\right) \pitchfork \alpha_{+}\right) e^{B} \alpha_{+}
$$


It follows that the composition in the statement is defined by

$$
\begin{aligned}
P S S \circ \Psi_{\tilde{g}} \circ & P S S\left(\alpha_{-}\right)=\sum_{\tilde{l}} \#\left(\mathrm{ev}_{-} \mathcal{M}\left(P_{-}, \tilde{l}\right) \pitchfork \alpha_{-}\right) P S S\left(\tilde{l}^{g}\right) \\
& =\sum_{\tilde{l}, B, \alpha_{+}} \#\left(\mathrm{ev}_{-} \mathcal{M}\left(P_{-}, \tilde{l}\right) \pitchfork \alpha_{-}\right) \#\left(\mathrm{ev}_{+} \mathcal{M}\left(P_{+}, \tilde{l}^{g} \# B\right) \pitchfork \alpha_{+}\right) e^{B} \alpha_{+} \\
& =\sum_{\alpha_{+}, B} \#\left(\left(\mathrm{ev}-\times \mathrm{ev}_{+}\right) \mathcal{M}\left(P_{g}(R), \sigma_{w}^{g} \# B\right) \pitchfork\left(\alpha_{-} \times \alpha_{+}\right)\right) e^{B} \alpha_{+}
\end{aligned}
$$

where the last equality uses the isomorphism of the moduli spaces via gluing, and $\sigma_{w}^{g}$ can be formally written as $w^{g} \#(-w)$. Here the notation $\mathcal{M}\left(P_{g}(R), \sigma\right)$ denotes the subset of elements in $\mathcal{M}\left(P_{g}(R)\right)$ that represent the section class $\sigma$.

Comparing with the expression for $\Psi_{L}\left(g, \sigma_{0}\right)$, we see that the only thing left to show is that $w^{g} \#(-w)$ belongs to the same section class for any $\tilde{l}=[l, w]$. Then take $\sigma_{0}$ to be the common class and the proposition is established. This we show in the next lemma.

Lemma 4.2 Let $\tilde{l}=[l, w] \in \widetilde{P}_{L} M$ and $\widetilde{g} \in \widetilde{\mathcal{P}}_{L} \operatorname{Ham}(M, \omega)$. The equivalence class of the section $\sigma_{w}^{g}=w \#\left(-w^{g}\right)$ of $P(R)$ defined above does not depend on $w$.

Proof We show that the sections $\sigma_{w}^{g}$ are homotopic, which implies equivalence. Suppose that $(g, \widetilde{g})=$ id then the resulting bundle $\left(P_{g}, N\right)$ is the trivial bundle pair. Let $p r:\left(P_{g}, N\right) \rightarrow(M, L)$ be the projection to the fiber, then $p r(w \#(-w))$ represents 0 in $\pi_{2}(M, L)$. In particular, $w \#(-w)$ is homotopic to the section $D^{2} \times\{l(0)\}$. Thus, the equivalence class of $w^{g} \#(-w)$ does not depend on $w$.

Let $\tilde{p}=[p, p]$ be the trivial path and lifting to $\widetilde{P}_{L} M$ where $p \in L$. Then $\sigma_{p}^{g}=$ $w_{p}^{g} \#(-p)$. Choose a path $\left\{p_{t}\right\}$ on $L$ which connects $p$ and $p^{\prime}$, then it is obvious that $\sigma_{p}^{g}$ and $\sigma_{p^{\prime}}^{g}$ are homotopic through sections with boundary on $N$ by $\sigma_{p_{t}}^{g}$. It follows that the equivalence class of $\sigma_{p}^{g}$ does not depend on the point $p$.

For general $[l, w]$ we show that it is the combination of the above two special cases. First, $(g, \tilde{g})$ may be reparametrized such that $g_{t}=g_{1}$ for $t>\frac{1}{3}$, and $w_{p}^{g}$ maps $D_{+} \cap\left\{\Im z<\frac{2}{3}\right\}$ to $g_{1}(p)$ for all $p \in L$. Then, $w$ may be reparametrized such that $w=l(0)$ on $D_{+} \cap\left\{\Im z>\frac{1}{3}\right\}$. The resulting section $\sigma_{w}^{g}$ is homotopic to the original $w^{g} \#(-w)$.

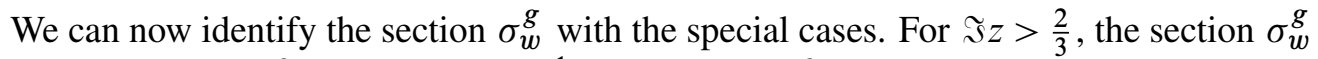
coincides with $\sigma_{l(0)}^{g}$ and for $\mathfrak{I}_{g} z \frac{1}{3}$, the section $\sigma_{w}^{g}$ coincides with $g_{1} \circ(w \#(-w))$. For $\frac{1}{3}<\Im z<\frac{2}{3}$, the section $\sigma_{w}^{g}$ coincides with $g_{1}(l(0))$. 
Similar construction for another element $\left[l^{\prime}, w^{\prime}\right]$ gives a section $\sigma_{w^{\prime}}^{g}$. Now the homotopies described in the special cases define a homotopy between the sections $\sigma_{w}^{g}$ and $\sigma_{w^{\prime}}^{g}$

\section{A second proof of Theorem 1.1}

We now present another proof of the main theorem based on the analytic Seidel map which is proved to be trivial in the symplectically aspherical case, thanks to additional algebraic structures and ideas appearing in Leclercq [10]. This algebraic proof, which is geometrically less meaningful, is conceptually more elementary. As above, we work with coefficients in $\mathbb{Z}_{2}$ and we assume the pair $(M, L)$ to be symplectically aspherical. In that case, the proofs of the intermediate steps are fully written in [10]. Moreover, this proof does not extend as it is to integer and rational coefficients (see Section A.8 for some details).

Recall that since we work under the symplectic asphericity condition, the quantum homology of $L$ is actually its Morse homology and we may forget about the Novikov ring on the Floer side.

First, notice that there is an "instantaneous" version of the analytic Seidel map described in Section 4, consisting in using, instead of an isotopy $g \in \mathcal{P}_{L} \operatorname{Ham}(M, \omega)$, only a Hamiltonian diffeomorphism preserving $L, g_{1} \in \operatorname{Ham}_{L}(M, \omega)$. We let $\Psi_{L}^{i}\left(g_{1}\right)$ denote this instantaneous version, as well as its Morse counterpart.

Indeed, for any diffeomorphism $h \in \operatorname{Diff}(L)$, we can consider the morphism $\Psi_{L}^{i}(h)$ identifying the complexes $M C_{*}(L ; f, \rho)$ and $M C_{*}\left(L ; f^{h}, \rho^{h}\right)$, where $f^{h}=f \circ h^{-1}$ and $\rho^{h}=\left(h^{-1}\right)^{*} \rho$, via the following equivalences:

$$
x \in \mathrm{Crit}_{k}(f) \Longleftrightarrow x^{h}=h(x) \in \mathrm{Crit}_{k}\left(f^{h}\right)
$$

$\gamma$ flow line of $(f, \rho) \Longleftrightarrow \gamma^{h}=h \circ \gamma$ flow line of $\left(f^{h}, \rho^{h}\right)$

This identification induces an isomorphism on homology which commutes with the usual comparison morphism. (The commutativity can be obtained at the chain level, by choosing a regular homotopy $(\boldsymbol{f}, \boldsymbol{\rho})$ between $\left(f_{0}, \rho_{0}\right)$ and $\left(f_{1}, \rho_{1}\right)$ and on the other side $\left(f^{h}, \rho^{h}\right)$.) The action of $h$ on the (Morse) homology of $L$ can then be seen as the composition of this identification with the usual Morse comparison morphism (in order to end up in the initial complex $\left.M C_{*}(L ; f, \rho)\right)$.

Now, if we choose $g_{1} \in \operatorname{Ham}_{L}(M, \omega)$, we can compare the Floer and Morse instantaneous Seidel maps respectively associated to $g_{1}$ and its restriction to $L$. The first step of this second proof is to show that they do coincide, via the Lagrangian PSS morphism. 
Lemma 5.1 The following diagram commutes in homology:

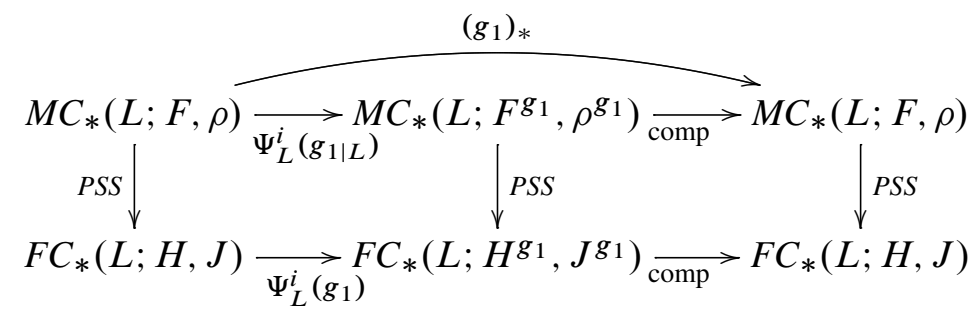

Proof Since the PSS morphism commutes with the classical Morse and Floer comparison morphisms (see, for example, Leclercq [10, Lemma 2.3] for a proof), the right square of (5-1) commutes in homology.

Now the left square commutes (at the chain level) for any regular choices of Floer and Morse data on the left and their respective pullbacks via the Hamiltonian diffeomorphism and its restriction to $L$. Indeed, in that case, there is an identification of the moduli spaces defining the involved PSS morphisms.

Since (5-1) commutes, it suffices to prove that the composition of the two morphisms at the Floer level induces the identity in homology. In order to do so, we express the action of $\Psi_{L}^{i}\left(g_{1}\right)$ on $F H_{*}(L)$ in terms of the (non-instantaneous) analytic Seidel map (and Poincaré duality).

Lemma 5.2 The following diagram commutes:

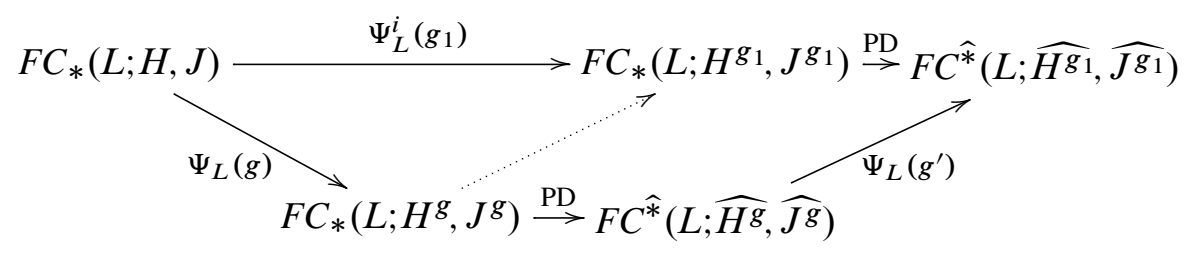

where $g^{\prime}$ is the isotopy defined as $g_{t}^{\prime}=g_{1} \circ g_{1-t}^{-1}$. (The dotted arrow, usually denoted $\left[\Psi_{L}\left(g^{\prime}\right)\right]_{!}$, is defined by the commutativity of the right square, that is, by pre- and post-composing $\Psi_{L}\left(g^{\prime}\right)$ with Poincaré duality.)

Let us briefly recall the Floer theoretic version of Poincare duality. This isomorphism is defined by identifying the complexes $F C(L ; H, J)$ and $F C(L ; \hat{H}, \widehat{J})$ for any regular pair $(H, J)$, where $(\hat{H}, \widehat{J})$ is "dual" to $(H, J)$, that is, is defined as $\hat{H}_{t}(x)=-H_{1-t}(x)$ and $\widehat{J}_{t}=J_{1-t}$. The generators are geometrically the same orbits but considered with the opposite orientation, and so are the half-tubes defining the respective differentials. (A priori $\widehat{*}=-*$, if the references of the Maslov indices are 
chosen so that they geometrically coincide. Other choices amount to global shifts of the degree which do not matter here.)

Via straightforward computations, it is easy to see that $\left(\widehat{H^{g}}\right)^{g^{\prime}}={\widehat{H^{g}}}^{1}=\widehat{H}^{g_{1}}$ and $\left(\widehat{J^{g}}\right)^{g^{\prime}}=\widehat{J^{g_{1}}}=\widehat{J}^{g_{1}}$, and that all the involved pairs are regular if and only if $(H, J)$ is. Thus, (5-2) makes sense as it is (on the complexes).

Remark 5.3 Notice for later use, that Poincare duality commutes with the usual comparison morphism of Floer homology, since it even commutes at the chain level as soon as one uses timewise dual homotopies.

Proof of Lemma 5.2 Figure 1 illustrates the evolution of an orbit along (5-2).

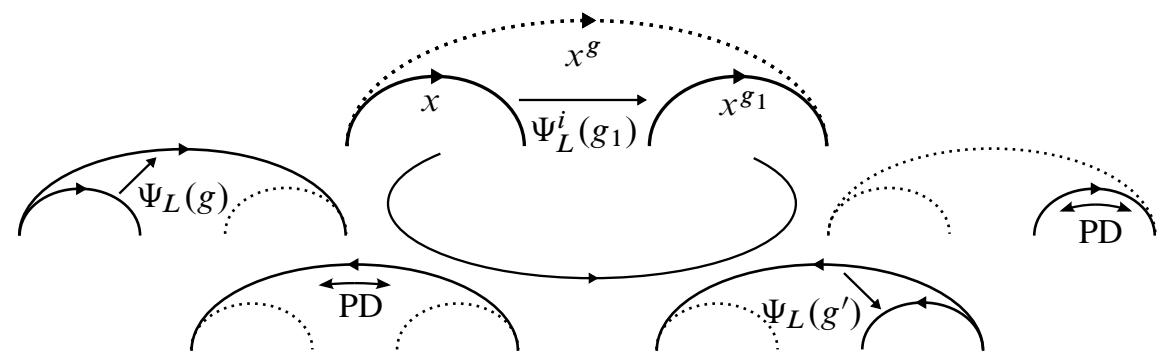

Namely, for each time $t$, we have:

$$
\begin{aligned}
x(t) & \rightsquigarrow x^{g}(t)=g_{t}(x(t)) \\
& \rightsquigarrow \mathrm{PD}\left(x^{g}\right)(t)=g_{1-t}(x(1-t)) \\
& \rightsquigarrow\left(\mathrm{PD}\left(x^{g}\right)\right)^{g^{\prime}}(t)=g_{1}(x(1-t)) \\
& \rightsquigarrow \mathrm{PD}^{-1}\left[\left(\mathrm{PD}\left(x^{g}\right)\right)^{g^{\prime}}\right](t)=g_{1}(x(t))=x^{g_{1}}(t)
\end{aligned}
$$

The half-tubes defining Floer differential evolve in a similar way and the commutativity of the diagram immediately follows.

Now comes the crucial point: When $(M, L)$ is symplectically aspherical, the analytic Seidel map acts trivially on (Morse) homology.

Lemma 5.4 The analytic Seidel map (of Proposition 4.1), is the identity, that is, $\Psi_{L}(g)$ acts on Floer homology as the usual comparison morphism.

Remark 5.5 Recall that the PSS morphism and $\Psi_{L}(g)$ can be composed since the action of $g$ preserves the component of $\mathcal{P}(L)$ consisting of contractible (in $\pi_{1}(M, L)$ ) paths (see Bialy-Polterovich [2, Theorem 1.8] for the proof of this result in the weakly exact case). 
The proof of the theorem now easily follows. Indeed, Lemma 5.4 allows us to replace the Seidel map by the usual comparison morphism, in the diagram induced in homology by (5-2). Thus, we get the commutative diagram

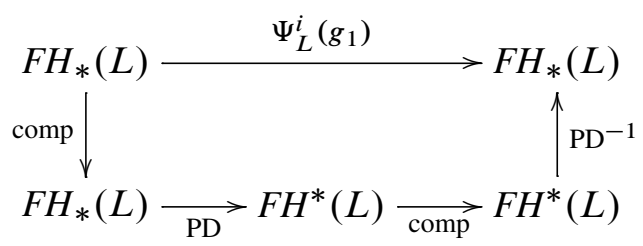

Now, as noticed above (see Remark 5.3), the comparison morphism commutes with Poincaré duality, thus we can permute the two morphisms composing the bottom line of (5-3). Since the Floer comparison morphism is natural, this shows that $\Psi_{L}^{i}\left(g_{1}\right)$ acts on $F H_{*}(L)$ as the comparison morphism and this, in turn, proves that the bottom line of (5-1) induces the identity in homology, which concludes the proof of Theorem 1.1.

Therefore, it only remains to prove Lemma 5.4. The fact that, for a symplectically aspherical pair $(M, L), \Psi_{L}(g)$ acts on Floer homology as the comparison morphism can be indirectly deduced from the commutative diagram of Leclercq [10, Proposition 3.1], by first arbitrarily "cutting" in two parts the Hamiltonian isotopy which we consider. However, the proof of [10, Proposition 3.1] itself can be easily adapted to immediately show that the diagram

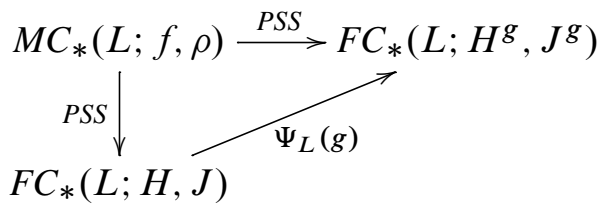

commutes in homology, and this commutativity amounts to the triviality of the analytic Seidel map (compare with Proposition 4.1).

Proof of Lemma 5.4 We know from Leclercq [10, Sections 3.1-3.2] that all the involved groups are $M H_{*}(L)$-modules, and that this additional structure is preserved by the PSS morphism and by $\Psi_{L}(g)$. Thus $\Phi=(P S S)^{-1} \circ \Psi_{L}(g) \circ P S S$ is an endomorphism of $M H_{*}(L)$ (as a module over itself).

Now $\Phi([L])=[L]$, since $[L]$ generates $M H_{\text {top }}(L)$ (due to $\mathbb{Z}_{2}$ coefficients, signs are arbitrary). Since $[L]$ is also the unit of the $\operatorname{ring}\left(M H_{*}(L), \cdot\right)$, we have for any $a \in M H_{*}(L)$ :

$$
\Phi(a)=\Phi(a \cdot[L])=a \cdot \Phi([L])=a \cdot[L]=a .
$$


Thus $\Phi$ is the identity and (5-4) commutes. Now, since the PSS morphism commutes with the usual comparison morphisms, the composition of the PSS morphisms in (5-4) is nothing but the Floer comparison morphism. Thus both morphisms

$$
\Psi_{L}(g), \text { comp: } F C_{*}(L ; H, J) \longrightarrow F C_{*}\left(L ; H^{g}, J^{g}\right)
$$

coincide in homology.

\section{Appendix A Orientation of the moduli spaces}

We are going to follow closely the orientation conventions of Biran-Cornea [3, Appendix] (also compare with Fukaya-Oh-Ohta-Ono [6, Section 44]) and describe the orientations for the various moduli spaces used in the main text. In this appendix, we adapt to coefficients $\mathbb{Z}$ or $\mathbb{Q}$ the various definitions and propositions for proving the main theorem, when the pair $(M, L)$ has a relatively spin structure and the monodromy preserves the relatively spin structure. We will work in the case where $(M, L)$ is weakly exact. In particular, there is no non-trivial holomorphic discs with boundaries on $L$ and $\partial_{\text {Pearl }}=\partial_{\text {Morse }}$.

\section{A.1 Half dises}

Let $(M, L)$ be a pair of symplectic manifold and Lagrangian submanifold. Let $\sigma_{M}^{\prime}$ denote a triangulation on $M$ such that $L$ is a subcomplex. The induced triangulation on $L$ is denoted $\sigma_{L}^{\prime}$. We say that $(M, L)$ is relatively spin with a relatively spin structure $\mathfrak{S}_{r}$ (with respect to the triangulation $\sigma_{M}^{\prime}$ ), if

(1) There is $\alpha \in H^{2}\left(M, \mathbb{Z}_{2}\right)$ such that $\left.\alpha\right|_{L}=w_{2}(T L)$

(2) There is an oriented vector bundle $V^{\prime} \rightarrow M_{(3)}^{\prime}$, the 3-skeleton of $\sigma_{M}^{\prime}$, so that $w_{2}\left(V^{\prime}\right)=\alpha$, and

(3) an extension of the trivialization of $V^{\prime} \oplus T L$ on $L_{(1)}^{\prime}$ onto $L_{(2)}^{\prime}$, the 1 - and 2-skeleton of $\sigma_{L}^{\prime}$ respectively.

In fact, once there is an oriented vector bundle $V^{\prime} \rightarrow M_{(3)}^{\prime}$ satisfying (3) above, we have $\left.w_{2}\left(V^{\prime}\right)\right|_{L}=w_{2}(T L)$. It is shown in [6, Section 44] that the moduli spaces of (parametrized) pseudo-holomorphic discs in $M$ with boundaries on $L$ can be coherently oriented when $(M, L)$ is relatively spin.

Let $\left(\mathbf{H}_{ \pm}, \mathbf{J}_{ \pm}\right)$be a $C^{\infty} z$-dependent ( $z \in Z_{ \pm}$, see Section 4.1) Hamiltonian function and a compatible almost complex structure on $M$, such that

- $\left.\left(\mathbf{H}_{ \pm}, \mathbf{J}_{ \pm}\right)\right|_{z \in D_{ \pm}^{2}}=\left(0, J_{0}\right)$ and 
- $\left.\left(\mathbf{H}_{ \pm}, \mathbf{J}_{ \pm}\right)\right|_{ \pm \Re z>1}=(H, J)$.

For $\tilde{l}=[l, w]$, let $\mathcal{M}_{-}\left(M, L ; \mathbf{H}_{-}, \mathbf{J}_{-} ; \tilde{l}\right)$ denote the moduli space of perturbed half discs $u_{-}: Z_{-} \rightarrow M$, which are finite energy solutions to (4-2), such that $u$ converges to $l$ as $s \rightarrow \infty$ and $I_{\mu}\left(w \# u_{-}\right)=0$. Similarly, for $\widetilde{l}^{\prime}=\left[l, w^{\prime}\right]$ there is the moduli space $\mathcal{M}_{+}\left(M, L ; \mathbf{H}_{+}, \mathbf{J}_{+} ; \widetilde{l}^{\prime}\right)$, which consists of perturbed half discs $u_{+}: Z_{+} \rightarrow M$, such that $u_{+}$converges to $l$ as $s \rightarrow-\infty$ and $I_{\mu}\left(w^{\prime} \#\left(-u_{+}\right)\right)=0$. Following the gluing argument described in Proposition 4.1, let

$Z(R)=\left(Z_{-} \backslash(R+1, \infty) \times[0,1]\right) \cup\left(Z_{+} \backslash(-\infty,-R-1) \times[0,1]\right) /(s, t) \sim(s-R-2, t)$

and $(\mathbf{H}(R), \mathbf{J}(R))$ be the glued data. Then $Z(R)$ is conformally equivalent to a disc and the glued equation is the pseudo-holomorphic equation with compact perturbation. Let $\mathcal{M}(M, L ; \mathbf{H}(R), \mathbf{J}(R))$ be the moduli space of the solutions (we also use $\mathcal{M}(M, L ; \mathbf{H}(R), \mathbf{J}(R) ; B)$ where $B=\left[w^{\prime} \#(-w)\right]$ is the class represented by the maps), then it admits coherent orientation from the relatively spin structure on $L$. The gluing map is a local diffeomorphism:

$(\mathrm{A}-1) \mathcal{M}_{-}\left(M, L ; \mathbf{H}_{-}, \mathbf{J}_{-} ; \widetilde{l}\right) \times \mathcal{M}_{+}\left(M, L ; \mathbf{H}_{+}, \mathbf{J}_{+} ; \widetilde{l}^{\prime}\right) \rightarrow \mathcal{M}(M, L ; \mathbf{H}(R), \mathbf{J}(R))$

We determine the orientations of the spaces $\mathcal{M}_{ \pm}\left(M, L ; \mathbf{H}_{ \pm}, \mathbf{J}_{ \pm} ; \widetilde{l}\right)$, with the chosen relatively spin structure, in the following steps:

- Fix $\tilde{l}=[l, w]$. Choose an orientation of $\mathcal{M}_{-}\left(M, L ; \mathbf{H}_{-}, \mathbf{J}_{-} ; \tilde{l}\right)$. The moduli spaces $\mathcal{M}(M, L ; \mathbf{H}(R), \mathbf{J}(R))$ on the right of (A-1) are coherently oriented by the chosen relatively spin structure, for any $\widetilde{l}^{\prime}=\left[l, w^{\prime}\right]$. Thus, the condition that (A-1) is orientation preserving induces coherent orientations for all moduli spaces of the type $\mathcal{M}_{+}\left(M, L ; \mathbf{H}_{+}, \mathbf{J}_{+} ; \widetilde{l}^{\prime}\right)$, where $\widetilde{l}^{\prime}=\left[l, w^{\prime}\right]$.

- For each Hamiltonian path $l$, choose $\tilde{l}=[l, w]$ and an orientation of the moduli space $\mathcal{M}_{-}\left(M, L ; \mathbf{H}_{-}, \mathbf{J}_{-} ; \widetilde{l}\right)$. The step above determines coherent orientations for all moduli spaces of the type $\mathcal{M}_{+}\left(M, L ; \mathbf{H}_{+}, \mathbf{J}_{+} ; \tilde{l}^{\prime}\right)$.

- We note the following special case of (A-1):

$$
\mathcal{M}_{-}\left(M, L ; \mathbf{H}_{-}, \mathbf{J}_{-} ; \widetilde{l}\right) \times \mathcal{M}_{+}\left(M, L ; \mathbf{H}_{+}, \mathbf{J}_{+} ; \widetilde{l}\right) \rightarrow \mathcal{M}(M, L ; \mathbf{H}(R), \mathbf{J}(R) ; 0) \cong L
$$

Again, the condition that (A-1) preserves orientation induces coherent orientation of all moduli spaces of the type $\mathcal{M}_{-}\left(M, L ; \mathbf{H}_{-}, \mathbf{J}_{-} ; \tilde{l}^{\prime}\right)$.

\section{A.2 Floer homology}

Let $\widetilde{\mathcal{M}}\left(M, L ; H, J ; l_{-}, l_{+}\right)$be the moduli space of the parametrized finite energy solutions to the equation (4-1), such that as $s \rightarrow \pm \infty, u \rightarrow l_{ \pm}$. Let $\widetilde{l}_{-}=\left[l_{-}, w_{-}\right]$and 
$\tilde{l}_{+}=\left[l_{+}, w_{+}\right]$. By an abuse of notations, we use $\widetilde{\mathcal{M}}\left(M, L ; H, J ; \tilde{l}_{-}, \tilde{l}_{+}\right)$to denote the subspace of solutions $u$ which satisfy $I_{\mu}\left(w_{-} \# u \# w_{+}\right)=0$. Then the orientation of $\widetilde{\mathcal{M}}\left(M, L ; H, J ; \tilde{l}_{-}, \tilde{l}_{+}\right)$is determined by requiring the gluing map below to preserve the orientations:

$$
\begin{aligned}
\mathcal{M}_{-}\left(M, L ; \mathbf{H}_{-}, \mathbf{J}_{-} ; \tilde{l}_{-}\right) \times \widetilde{\mathcal{M}}\left(M, L ; H, J ; \tilde{l}_{-}, \tilde{l}_{+}\right) \times \mathcal{M}_{+}(M, L ; & \left.\mathbf{H}_{+}, \mathbf{J}_{+} ; \tilde{l}_{+}\right) \\
& \rightarrow \mathcal{M}(M, L ; \mathbf{H}, \mathbf{J})
\end{aligned}
$$

That the above map is a local homeomorphism follows from similar arguments in [6, Section 44].

We will drop $(M, L ; H, J)$ from the notations in the following. To define the Floer homology, one considers the moduli space

$$
\mathcal{M}\left(l_{-}, l_{+}\right):=\widetilde{\mathcal{M}}\left(l_{-}, l_{+}\right) / \mathbb{R}
$$

of unparametrized solutions, where the action of $\alpha \in \mathbb{R}$ on $u: \mathbb{R} \times[0,1] \rightarrow M$ is given by

$$
(\alpha \circ u)(s, t)=u(s+\alpha, t)
$$

Then we require the following (locally defined homeomorphism) map to preserve the orientations:

$$
\mathcal{M}\left(l_{-}, l_{+}\right) \times \mathbb{R} \rightarrow \widetilde{\mathcal{M}}\left(l_{-}, l_{+}\right)
$$

Then the Floer boundary map is defined by

$$
\partial_{\text {Floer }} \tilde{l}_{-}:=\sum_{\tilde{l}_{+}} \# \mathcal{M}\left(\tilde{l}_{-}, \tilde{l}_{+}\right) \tilde{l}_{+}
$$

where the sum is along all $\tilde{l}_{+}$such that the moduli space involved has dimension 0 . By the conventions we adopt, the following gluing map preserves the orientations:

$$
\widetilde{\mathcal{M}}\left(\tilde{l}_{-}, \tilde{l}_{0}\right) \times \widetilde{\mathcal{M}}\left(\tilde{l}_{0}, \tilde{l}_{+}\right) \rightarrow \widetilde{\mathcal{M}}\left(\tilde{l}_{-}, \tilde{l}_{+}\right)
$$

Passing to the unparametrized moduli spaces, it induces the inclusion as boundaries:

$$
\mathcal{M}\left(\tilde{l}_{-}, \tilde{l}_{0}\right) \times \mathcal{M}\left(\tilde{l}_{0}, \tilde{l}_{+}\right) \rightarrow \partial \overline{\mathcal{M}\left(\tilde{l}_{-}, \tilde{l}_{+}\right)}
$$

where the orientation is given by $(-1)^{\operatorname{dim} \mathcal{M}\left(\tilde{l}_{-}, \tilde{l}_{0}\right)}$. It then follows that $\partial_{\text {Floer }}^{2}=0$ over $\mathbb{Z}$ or $\mathbb{Q}$. 


\section{A.3 PSS maps}

We refer to the main text, (4-3) and (4-4), for the definition of the PSS maps that relate the quantum and Floer homologies of $(M, L)$. Here we describe the orientations involved. The coefficient in (4-3) is oriented as the fiber product

$$
\alpha_{-} \times_{\mathrm{ev}_{-}} \mathcal{M}\left(P_{-}, \widetilde{l}\right)
$$

and the coefficient in (4-4) is oriented as the fiber product

$$
\mathcal{M}\left(P_{+}, \tilde{l}^{g} \# B\right) \mathrm{ev}_{+} \times_{L} \alpha_{+}
$$

Using the conventions established in Biran-Cornea [3, Appendix A.1] and the previous subsections, these define chain maps in $\mathbb{Z}$ or $\mathbb{Q}$. The orientations here together with (A-2) (and the arguments that follow) imply that (4-5) holds in $\mathbb{Z}$ or $\mathbb{Q}$.

\section{A.4 Geometric Seidel map}

A smooth map $f:(M, L) \rightarrow(M, L)$ preserves $\mathfrak{S}_{r}$ if:

(1) There is a common refinement $\sigma_{M}$ of the triangulations $\sigma_{M}^{\prime}$ and $f_{*} \sigma_{M}^{\prime}$ on $M$

(2) and a common extension $V \rightarrow M_{(3)}$ of the vector bundles $V^{\prime}$ and $f_{*} V^{\prime}$

(3) as well as a trivialization of $V \oplus T L$ on $L_{(2)}$, which is a common extension of the trivializations of $V^{\prime} \oplus T L$ and $f_{*}\left(V^{\prime} \oplus T L\right)$ on the respective $1-$ and 2-skeletons.

We use $W_{f}^{u}(x)$ (respectively $\left.W_{f}^{s}(x)\right)$ to denote the unstable (respectively stable) manifold (under the negative gradient flow) of the critical point $x$ of a Morse function $f$.

Proposition A.1 Suppose that $L \subset M$ is a relatively spin monotone Lagrangian submanifold, with minimal Maslov index 2 and let a fixed relatively spin structure $\mathfrak{S}_{r}$ be given. Suppose that $g_{1}:(M, L) \rightarrow(M, L)$ preserves $\mathfrak{S}_{r}$. Then $\Psi_{L}\left(g, \sigma_{0}\right)$ (see Definition 2.5) can be defined over $\mathbb{Z}$ or $\mathbb{Q}$.

Proof We show that the moduli spaces of the type $\mathcal{M}\left(P_{g}, N ; \sigma ; F, G, J_{P} ; x_{-}, y_{+}\right)$ admit coherent orientations, from which the statement follows.

Let us start by showing that the moduli space of holomorphic discs representing the section class $\sigma_{0}$ as defined in Lemma 4.2 admits a coherent orientation. Notice that, for example, $\mathcal{M}\left(P_{-}, \widetilde{l}\right)$ is identified as $\mathcal{M}_{-}(\widetilde{l})$ and $\mathcal{M}\left(P_{+}, \widetilde{l^{g}}\right)$ is identified as $\mathcal{M}_{+}\left(\widetilde{l^{g}}\right)$, whose orientations are given as in Section A.1. The gluing construction in the proof 
of Proposition 4.1 implies that $\mathcal{M}\left(P_{g}, \sigma_{0}\right)$ admits coherent orientation such that the gluing map is orientation preserving:

$$
\mathcal{M}\left(P_{-}, \tilde{l}\right) \times \mathcal{M}\left(P_{+}, \tilde{l g}\right) \rightarrow \mathcal{M}\left(P_{g}(R), \sigma_{0}\right)
$$

Next, we show that the moduli spaces of holomorphic discs in $\left(P_{g}, N\right)$ representing the fiber classes are coherently oriented. Again, the moduli spaces might be empty, nonetheless, the index of the linearized operator can be oriented as described below. Consider $M \times I$ together with the product triangulation $\sigma_{\pi}^{\prime}$ induced from $\sigma_{M}^{\prime}$. We have $(M \times I)^{\prime}{ }_{3)} \subset M_{(3)}^{\prime} \times I$. Thus, the vector bundle $V^{\prime}$ induces a vector bundle $V_{\pi}^{\prime}$ on $(M \times I)_{(3)}^{\prime}$ by pulling back. Let $\sigma_{g}^{\prime}=g\left(\sigma_{\pi}^{\prime}\right)$ and $V_{g}^{\prime}=g_{*}\left(V_{\pi}^{\prime}\right)$, then

$$
\left.\left(\sigma_{g}^{\prime}, V_{g}^{\prime}\right)\right|_{M \times\{0\}}=\left(\sigma_{M}^{\prime}, V^{\prime}\right) \text { and }\left.\left(\sigma_{g}^{\prime}, V_{g}^{\prime}\right)\right|_{M \times\{1\}}=\left(g_{1 *} \sigma_{M}^{\prime}, g_{1 *} V^{\prime}\right)
$$

Since $g_{1}$ preserves $\mathfrak{S}_{r}$, there is a common refinement $\sigma_{M}$ of $\sigma_{M}^{\prime}$ and $g_{1 *} \sigma_{M}^{\prime}$ together with common extension $V$ of $V^{\prime}$ and $g_{1 *} V^{\prime}$. The mapping torus $M \times I /(x, 0) \sim$ $\left(g_{1}(x), 1\right)$ is diffeomorphic to $M \times S^{1}$. Then the triangulations $\sigma_{g}^{\prime}$ on $M \times I$ and $\sigma_{M}$ on $M \times\{0\}$ define then a cellular decomposition $\sigma_{S^{1}}$ of $M \times S^{1}=\left.P_{g}\right|_{S^{1}} \subset P_{g}$. Similarly the vector bundles $V_{g}^{\prime}$ on $(M \times I)_{(3)}$ and $V$ on $M_{(3)}$ define a vector bundle $V_{S^{1}}$ on the 3 -skeleton $\left(M \times S^{1}\right)_{(3)}$ of the cellular decomposition $\sigma_{S^{1}}$. Using the presentation of $N$ as a mapping torus of $g_{1}$ given in Lemma 2.4, $N \subset P_{g}$ is a subcomplex in the cellular decomposition $\sigma_{S^{1}}$ and we denote the induced cellular decomposition $\sigma_{N}$. By construction, the trivialization of $V \oplus T L$ on $L_{(2)}$ induces a trivialization of $V_{S^{1}} \oplus T N=V_{S^{1}} \oplus T^{v} N \oplus \mathbb{R}$ on $N_{(2)}$, the 2-skeleton of $N$ in the cellular decomposition $\sigma_{N}$. In the construction of the geometric Seidel map, the holomorphic discs representing the fiber classes lie completely in $\left.P_{g}\right|_{S^{1}}$. Thus, the same arguments that define a coherent orientation of moduli spaces of holomorphic discs from a relatively spin structure (see, for example, Fukaya-Oh-Ohta-Ono [6, Section 44]) can be adapted here to give coherent orientations for the corresponding moduli spaces.

Lastly, by Lemma 2.2, any section class $\sigma$ differs from $\sigma_{0}$ by a fiber class. It follows from gluing theory that the coherent orientations of $\mathcal{M}\left(P_{g}, \sigma_{0}\right)$ and the fiber classes constructed above define coherent orientations for all the moduli spaces of the form $\mathcal{M}\left(P_{g}, \sigma\right)$.

Similar to the pearl complex (see Biran-Cornea [4; 3] and Cornea-Lalonde [5]), the moduli space $\mathcal{M}\left(P_{g}, N ; \sigma ; F, G, J_{P} ; x_{-}, y_{+}\right)$consists of multiple configurations of pseudo-holomorphic discs connected by Morse flow lines. Consider the configuration of a section connected to $x_{-}$and $y_{+}$by Morse flow lines, those of the other configurations and the compatibility among the configurations are similar to [3, Appendix A.2]. The 
corresponding moduli space is described by the following fiber product:

$$
W_{F}^{u}\left(x_{-}\right) \times_{N}\left(\mathcal{M}\left(P_{g}, \sigma\right) \times \mathbb{R}_{+}\right) \times_{N} W_{F}^{s}\left(y_{+}\right)
$$

The manifolds $W_{F}^{u}\left(x_{-}\right)$and $W_{F}^{s}\left(y_{+}\right)$are identified respectively with the manifolds $W_{f}^{u}(x)$ in $L_{-}$and $W_{f}^{s}(y)$ in $L_{+}$as oriented manifolds. $N$ is oriented as a fiber bundle over $S^{1}$, which is oriented as $\mathbb{R}^{1} / \mathbb{Z}$. The orientation of the fiber product follows from the convention as in [3]. Namely, locally it coincides with the orientation of $I \times N$ for some interval $I \subset \mathbb{R}$.

\section{A.5 The map $\Sigma\left(g, \sigma_{0}\right)$}

This map is used in the proof of Lemma 3.2. Its definition involves moduli spaces of the form $\mathcal{M}\left(P_{g}, N ; \sigma ; F, G, J_{P} ; y, z_{+}\right)$. For $y=y_{-}$, the orientation is provided in Proposition A.1, while for $y=y_{+}$, the orientation is given by a similar fiber product:

$$
W_{F}^{u}\left(y_{+}\right) \times_{N}\left(\mathcal{M}\left(P_{g}, \sigma\right) \times \mathbb{R}_{+}\right) \times_{N} W_{F}^{s}\left(z_{+}\right)
$$

The orientation of $W_{F}^{u}\left(y_{+}\right)$is induced by the orientation of $W_{F}^{s}\left(y_{+}\right)$and $N$. Then (3-1) with the product orientations gives the oriented boundary components.

\section{A.6 The map $\Phi_{ \pm}$}

These maps are the comparison maps for the quantum homology of $Q H_{*}(M, L)$, which are used in the proof of Lemma 2.7. With the weakly exact condition, the maps coincide with the comparison maps for the Morse theory of $L$ with coefficients in $\Lambda_{L}$. Let $\widetilde{L}_{ \pm}=L_{ \pm} \times[0,1]$ and $\widetilde{F}_{ \pm}=\left.\tilde{F}\right|_{\tilde{L}_{ \pm}}$, etc. The manifold $\widetilde{L}_{ \pm}$is oriented as fiber bundle over $[0,1]$, where $[0,1]$ carries the natural orientation. We identify $W_{\widetilde{F}_{ \pm}}^{s}\left(x_{0, \pm}\right)$ with $W_{f_{0, \pm}}^{s}(x)$ and $W_{\widetilde{F}_{ \pm}}^{u}\left(y_{1, \pm}\right)$ with $W_{f_{1, \pm}}^{u}(y)$ as oriented manifolds. The map $\Phi_{ \pm}$ is defined as

$$
\Phi_{ \pm}\left(x_{ \pm}\right):=\sum_{y_{ \pm}} \# \mathcal{M}_{\text {Morse }}\left(x_{0, \pm}, y_{1, \pm}\right) y_{ \pm}
$$

where the sum is along $y_{ \pm}$with $\left|y_{ \pm}\right|^{L}=\left|x_{ \pm}\right|^{L}$. The orientations of the moduli spaces in the definition are induced by the choice of the orientations of the stable and unstable manifolds.

\section{A.7 The map $\Sigma\left(\cdot ; \sigma_{0}\right)$}

This map is used in the proof of Lemma 2.7. We note that to adapt the proof of Lemma 2.7 to coefficient $\mathbb{Z}$ or $\mathbb{Q}$, we only need to show that in $\mathbb{Z}$ or $\mathbb{Q}$ :

(A-3) $\pm \Sigma\left(\cdot ; \sigma_{0}\right) \circ \partial_{\text {Pearl }} \pm \partial_{\text {Pearl }} \circ \Sigma\left(\cdot ; \sigma_{0}\right)=\Phi_{+} \circ \Psi_{L_{0}}\left(g, \sigma_{0}\right)-\Psi_{L_{1}}\left(g, \sigma_{0}\right) \circ \Phi_{-}$ 
Thus, we only need to verify the orientations of the moduli spaces involved on the right side. Since the signs on the left hand side would not affect our arguments in the proof of Lemma 2.7, we will not concern ourselves with them.

The map $\Sigma\left(\cdot ; \sigma_{0}\right)$ involves the moduli spaces of the form

$$
\mathcal{M}\left(\widetilde{P}, \tilde{N} ; \sigma ; \widetilde{F}, \widetilde{G}, \widetilde{J} ; x_{0,-}, y_{1,+}\right),
$$

whose orientation is given by the following fiber product:

$$
W_{\widetilde{F}}^{u}\left(x_{0,-}\right) \times_{\widetilde{N}}\left(\mathcal{M}(\widetilde{P}, \sigma) \times \mathbb{R}_{+}\right) \times_{\widetilde{N}} W_{\widetilde{F}}^{S}\left(y_{1,+}\right)
$$

The orientations of the spaces involved are as follows. The manifold $\tilde{N}$ is oriented as a fibration over $[0,1]$ with the natural orientation. We make the following identifications between various (un)stable manifolds as oriented manifolds:

- $W_{\widetilde{F}}^{s}\left(x_{0,+}\right)$ with $W_{f_{0,+}}^{s}(x)$,

- $W_{\widetilde{F}}^{u}\left(y_{1,-}\right)$ with $W_{f_{1,-}}^{s}(y)$,

- $W_{\widetilde{F}}^{s}\left(y_{1,+}\right)$ with $W_{\widetilde{F}_{+}}^{s}(y)$ and

- $W_{\widetilde{F}}^{u}\left(x_{0,-}\right)$ with $W_{\widetilde{F}_{0}}^{u}(x)$.

The orientations of the other (un)stable manifolds are induced from these choices. The moduli space $\mathcal{M}(\widetilde{P}, \sigma)$ consists of the pseudo-holomorphic sections in $P_{g} \times\{t\}$ for some $t \in[0,1]$, and it can be oriented as a fibration over $[0,1]$, with fiber $\mathcal{M}\left(P_{g}, \sigma\right)$.

Next we consider the boundary of the dimension 1 moduli space

$$
\mathcal{M}\left(\tilde{P}, \tilde{N} ; \sigma ; \widetilde{F}, \widetilde{G}, \widetilde{J} ; x_{0,-}, y_{1,+}\right) .
$$

We will concentrate on the boundary components that are involved in the right hand side of (A-3). Following the conventions in Biran-Cornea [3], the components are (the $(-1)^{\bullet}$ identifies the orientations)

$$
\begin{aligned}
(-1)^{n-\left|y_{1,+}\right|^{L}} W_{\widetilde{F}}^{u}\left(x_{0,-}\right) \times \times_{\widetilde{N}}(\mathcal{M} & \left.(\widetilde{P}, \sigma) \times \mathbb{R}_{+}\right) \\
& \times{ }_{\widetilde{N}}\left(\mathcal{M}_{\text {Morse }}^{\widetilde{N}}\left(y_{0,+}, y_{1,+}\right) \times W_{\widetilde{F}}^{s}\left(y_{0,+}\right)\right)
\end{aligned}
$$

and $\mathcal{M}_{\text {Morse }}^{\widetilde{N}}\left(x_{0,-}, x_{1,-}\right) \times W_{\widetilde{F}}^{u}\left(x_{1,-}\right) \times_{\widetilde{N}}\left(\mathcal{M}(\widetilde{P}, \sigma) \times \mathbb{R}_{+}\right) \times_{\widetilde{N}} W_{\widetilde{F}}^{s}\left(y_{1,+}\right)$.

The moduli spaces involved in the maps $\Phi_{+} \circ \Psi_{L_{0}}\left(g, \sigma_{0}\right)$ and $\Psi_{L_{1}}\left(g, \sigma_{0}\right) \circ \Phi_{-}$are respectively

$$
\mathcal{M}_{\text {Morse }}^{\widetilde{L}_{+}}\left(y_{0,+}, y_{1,+}\right) \times\left(W_{\widetilde{F}_{0}}^{u}\left(x_{0,-}\right) \times{ }_{N}\left(\mathcal{M}(P, \sigma) \times \mathbb{R}_{+}\right) \times_{N} W_{\widetilde{F}_{0}}^{s}\left(y_{0,+}\right)\right)
$$

and $\left(W_{\widetilde{F}_{1}}^{u}\left(x_{1,-}\right) \times_{N}\left(\mathcal{M}(P, \sigma) \times \mathbb{R}_{+}\right) \times{ }_{N} W_{\widetilde{F}_{0}}^{S}\left(y_{1,+}\right)\right) \times \mathcal{M}_{\text {Morse }}^{\widetilde{L}-}\left(x_{0,-}, x_{1,-}\right)$ 
where the Morse moduli spaces are of dimension 0 . To identify the orientations of the spaces in (A-4) and (A-5), we recall the following identity relating the fiber products with different bases:

$$
\begin{aligned}
(U \times \mathbb{R}) \times_{X \times \mathbb{R}}(V \times \mathbb{R})_{X \times \mathbb{R}} W & =(-1)^{|U|+|V|} U \times_{X} V \times_{X} W \\
\text { and } \quad U \times{ }_{X} \times \mathbb{R}(V \times \mathbb{R})_{X \times \mathbb{R}}(W \times \mathbb{R}) & =U \times_{X} V \times_{X} W
\end{aligned}
$$

where the transversality conditions are satisfied for all the fiber products, the product with $\mathbb{R}$ are oriented as fibrations over $\mathbb{R},|U|$ denotes the dimension of $U$, and $(-1)^{\bullet}$ identifies the orientation. It follows that the orientations of the first products in (A-4) and (A-5) are opposite and those of the second products coincide. Thus the right hand side of (A-3) is established in coefficient $\mathbb{Z}$ or $\mathbb{Q}$.

\section{A.8 Remarks concerning the algebraic proof}

Assume that the pair $(M, L)$ is relatively spin and consider integer or rational coefficients. Then diagrams (5-1) and (5-2) can still be defined (with Morse homology replaced by Lagrangian quantum homology) and still commute.

Diagram (5-1) By the details above, the morphisms which compose this diagram are well-defined (the only one which is not explicitly addressed above is the instantaneous version of Seidel's morphism, however, it is well-defined as the full Seidel map is, since we consider Hamiltonian diffeomorphisms which preserve the spin structure). The commutativity of the right-hand square also holds, up to homology (and states that the PSS morphism is well-defined). The commutativity of the left-hand square also holds at the chain level for relevant choices of the parameters.

Diagram (5-2) There are orientation conventions which make Poincaré duality welldefined in this case (see Biran-Cornea [3, Appendix A.2.6]). One can also check that this isomorphism commutes with the usual comparison morphism of Floer homology and that diagram (5-2) also commutes (at the chain level, for adequate choices of the parameters).

Obviously, Lemma 5.4 does not hold since (luckily enough) the relative Seidel morphism is not trivial. However, it should hold when we restrict ourselves to relatively aspherical pairs $(M, L)$ (regardless of the coefficient ring). What remains to be checked (in view of the paragraph above) for our algebraic proof to go through are the following steps:

(1) The Lagrangian quantum product is well-defined with unit $[L]$, the fundamental class of $L$ seen as a quantum homology class.

(2) Floer homology is a module over the Lagrangian quantum homology ring. 
(3) The PSS and Seidel morphisms are compatible with the module structures (the Lagrangian quantum homology ring being seen as a module over itself).

(4) $[L]$ is fixed via the composition of the PSS and Seidel morphisms (and finally $\left.P S S^{-1}\right)$.

Now, (1) holds (see [3, Appendix A.2.2]), as most probably (2) and (3). Concerning (4), the image of $[L]$ via the composition of these isomorphisms still has to generate the quantum homology module of degree $n$ of $L$, however, there is no a priori reason why it should coincide with $[L]$ (even with integer coefficients).

\section{References}

[1] M Akveld, D Salamon, Loops of Lagrangian submanifolds and pseudoholomorphic discs, Geom. Funct. Anal. 11 (2001) 609-650 MR1866797

[2] M Bialy, L Polterovich, Hamiltonian diffeomorphisms and Lagrangian distributions, Geom. Funct. Anal. 2 (1992) 173-210 MR1159829

[3] P Biran, O Cornea, Lagrangian topology and enumerative geometry arXiv: 1011.2271

[4] P Biran, O Cornea, Quantum structures for Lagrangian submanifolds arXiv: 0708.4221

[5] O Cornea, F Lalonde, Cluster homology arXiv:math. SG/0508345

[6] K Fukaya, Y-G Oh, K Ono, Lagrangian intersection Floer theory: anomaly and obstructions, preprint (2007)

[7] K Fukaya, P Seidel, I Smith, Exact Lagrangian submanifolds in simply-connected cotangent bundles, Invent. Math. 172 (2008) 1-27 MR2385665

[8] S Hu, F Lalonde, A relative Seidel morphism and the Albers map, Trans. Amer. Math. Soc. 362 (2010) 1135-1168 MR2563724

[9] F Lalonde, D McDuff, L Polterovich, Topological rigidity of Hamiltonian loops and quantum homology, Invent. Math. 135 (1999) 369-385 MR1666763

[10] R Leclercq, Spectral invariants in Lagrangian Floer theory, J. Mod. Dyn. 2 (2008) 249-286 MR2383268

[11] Y-G Oh, Relative Floer and quantum cohomology and the symplectic topology of Lagrangian submanifolds, from: "Contact and symplectic geometry (Cambridge, 1994)", Publ. Newton Inst. 8, Cambridge Univ. Press, Cambridge (1996) 201-267 MR1432465

[12] P Seidel, $\pi_{1}$ of symplectic automorphism groups and invertibles in quantum homology rings, Geom. Funct. Anal. 7 (1997) 1046-1095 MR1487754 
[13] M-L Yau, Monodromy and isotopy of monotone Lagrangian tori, Math. Res. Lett. 16 (2009) 531-541 MR2511631

SH: Department of Mathematics, Wilfrid Laurier University 75 University Ave. West, Waterloo, Ontario N2L 3C5, Canada

FL, RL: Département de mathématiques et de Statistique, Université de Montréal C.P. 6128, Succ. Centre-ville, Montréal, Québec H3C 3J7, Canada

shu@wlu.ca, lalonde@dms.umontreal.ca, leclercq@dms.umontreal.ca

Proposed: Leonid Polterovich

Seconded: Yasha Eliashberg, Simon Donaldson
Received: 4 March 2010

Revised: 30 June 2011 